\title{
Deep Equatorial Ocean Circulation Induced by a Forced-Dissipated Yanai Beam*
}

\author{
FRANÇOIS ASCANI AND ERIC FIRING \\ Department of Oceanography, University of Hawaii at Manoa, Honolulu, Hawaii \\ PIERRE DUTRIEUX \\ Physical Science Division, British Antarctic Survey, Cambridge, United Kingdom \\ JULIAN P. MCCREARY \\ Department of Oceanography, and International Pacific Research Center, University of Hawaii at Manoa, Honolulu, Hawaii \\ AKIO ISHIDA \\ Research Institute for Global Change, and Institute of Observational Research for Global Change, JAMSTEC, \\ Yokosuka, Japan
}

(Manuscript received 4 September 2009, in final form 23 December 2009)

\begin{abstract}
A complex pattern of zonal currents below the thermocline has been observed in the equatorial Pacific and Atlantic Oceans. The currents have typical speeds from 10 to $15 \mathrm{~cm} \mathrm{~s}^{-1}$ and extend as deep as $2500 \mathrm{~m}$. Their structure can be divided into two overlapping parts: the equatorial deep jets (EDJs), centered on the equator and alternating in the vertical with a wavelength of several hundred meters, and the Equatorial Intermediate Current system (EICS), composed of currents with large vertical scale and alternating with latitude over several degrees on either side of the equator. The strongest EICS current is a westward flow on the equator flanked by eastward currents at $2^{\circ} \mathrm{N}$ and $2^{\circ} \mathrm{S}$.

In the present study, the authors use idealized numerical simulations and analytical solutions to demonstrate that the EICS currents within $2.5^{\circ}$ from the equator could result from the self-advection with dissipation of a downward-propagating beam of monthly periodic Yanai (Rossby gravity) waves. The zonally restricted beam is generated in the eastern part of the basin by instabilities of the swift near-surface equatorial currents. For a weak Yanai wave amplitude and no dissipation, mean Eulerian currents resembling the three strongest EICS currents are obtained but only within the beam; in this case, the Eulerian flow is balanced by the waveinduced Stokes drift, yielding a zero-mean Lagrangian flow, and the water parcels conserve their potential vorticity (PV) and are stationary over a wave cycle. For larger amplitudes, the Yanai waves break, losing their energy to small vertical scales where it is dissipated. This dissipation changes the mean (wave averaged) PV of a water parcel within the beam, allowing the parcel to have a persistent equatorward drift across PV contours. This can be viewed as a wave-induced Sverdrup transport; by continuity and by virtue of the westward group velocity of long Rossby waves, this Lagrangian-mean meridional flow requires a Lagrangian-mean zonal flow within and to the west of the beam, with a meridional structure consistent with the three strongest EICS currents. This mechanism of EICS formation is active in some ocean general circulation models; its importance in the ocean remains to be evaluated.
\end{abstract}

\footnotetext{
* International Pacific Research Center Contribution Number 662 and School of Ocean and Earth Science and Technology Contribution Number 7869.

Corresponding author address: François Ascani, Department of Oceanography, University of Hawaii at Manoa, 1000 Pope Road, Honolulu, HI 96822.

E-mail: fascani@hawaii.edu
}

\section{Introduction}

The mean zonal currents below the thermocline down to about $2500 \mathrm{~m}$ in the Pacific and Atlantic oceans have a complex meridional and vertical structure (Fig. 1a; Firing 1987; Firing et al. 1998; Schott et al. 1995; Gouriou et al. 1999, 2001; Bourlès et al. 2002, 2003; Schott et al. 2003; Ollitrault et al. 2006). The currents may be divided into two sets. The first set, named the equatorial deep 
(a)

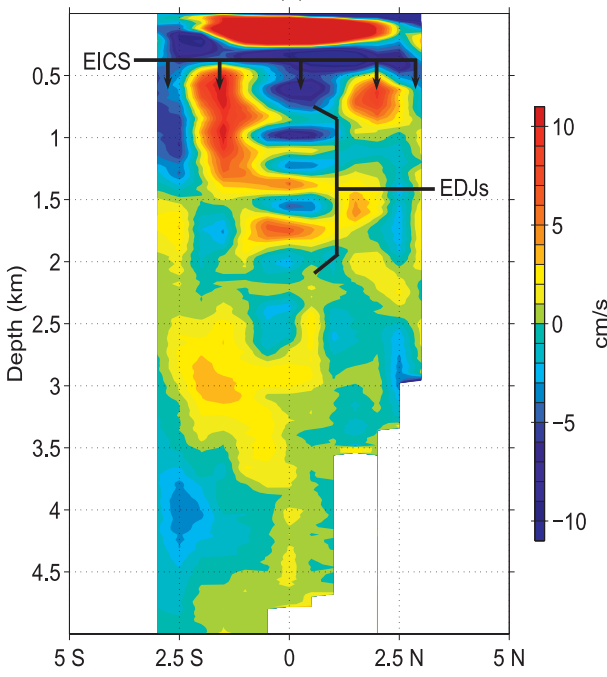

(c)

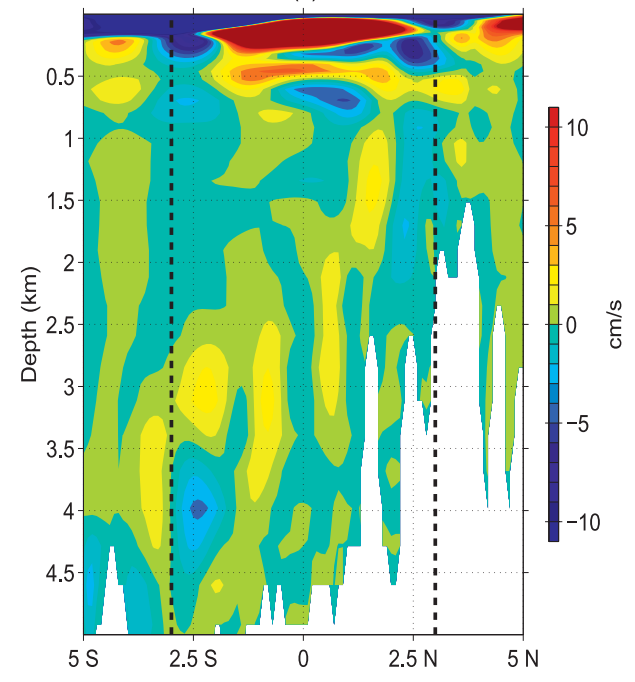

(b)

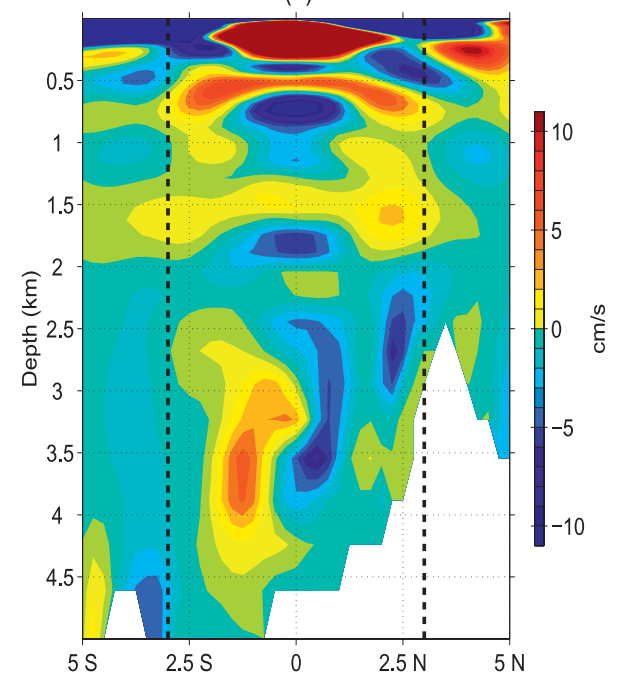

FIG. 1. Mean zonal velocity $u$ at $159^{\circ} \mathrm{W}$ from (a) observations taken during the Line Island Profiling Project (LIPP; Firing 1987), (b) years 20-22 of the I98 model, and (c) years 41-51 of the OFES model. The two runs are forced by climatological winds. The I 98 model has $1 / 4^{\circ}$ resolution in the horizontal and 55 levels, whereas OFES has $1 / 10^{\circ}$ resolution in the horizontal and 54 levels. Details of the runs can be found in I98 for their model and Masumoto et al. (2004) for OFES. jets (EDJs), is composed of currents centered near the equator and alternating in the vertical with a wavelength of several hundred meters. The second set is composed of currents with large vertical scale, alternating every $1^{\circ}-2^{\circ}$ in latitude. Although Gouriou et al. (2001) called them extra-equatorial jets, we prefer the alternative Equatorial Intermediate Current system (EICS) to explicitly include the predominantly westward flow on the equator and to give a sense that these currents form a closed circulation, as we demonstrate in the present paper.

The EICS currents have a mean speed of 10-15 $\mathrm{cm} \mathrm{s}^{-1}$, with a mean transport of 5-10 Sv (Firing 1987; Schott et al. 2003; Ollitrault et al. 2006; Brandt et al. 2008). They have been observed in synoptic sections across the Pacific and Atlantic Oceans (Firing et al. 1998; Gouriou et al. 2001). Recently, they have also been revealed in the mean zonal flow derived from various float programs (Ollitrault et al. 2006; Lebedev et al. 2007; Figs. 2, 3a). At $1000 \mathrm{~m}$, they are some of the strongest features of the deep ocean, second only to the deep subsurface flows found in the Southern Ocean and along midlatitude western boundaries. They appear as a set of eastward and westward currents within $10^{\circ}$ from the equator, zonally coherent over a large portion of each basin. In the Atlantic, the currents are found mainly in the western basin with the current axes in the Northern Hemisphere tilted equatorward to the east. In the Pacific, the amplitude and meridional positions of the currents stay remarkably constant over much of the basin width.

An EICS is present in high-resolution ocean general circulation models (OGCMs), such as the Ishida et al. (1998, hereafter I98) model and the OGCM for Earth 


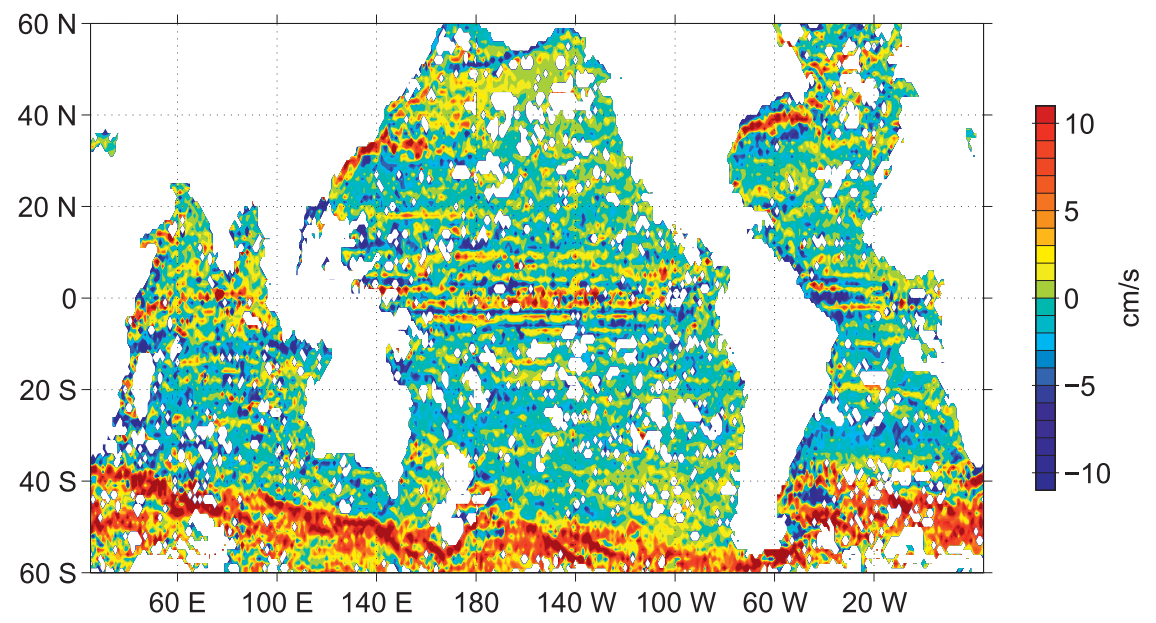

FIG. 2. Mean $u$ at $1000 \mathrm{~m}$ deduced from Argo floats (Lebedev et al. 2007). The map has been constructed by bin averaging over a $1^{\circ} \times 1^{\circ}$ grid. The average number of estimates per bin is about 10. White bins have no observations. Estimated errors reach $<0.5 \mathrm{~cm} \mathrm{~s}^{-1}$ near the equator.

Simulator (OFES; Masumoto et al. 2004), but with substantial differences between these models, and between models and observations. Although the meridional structure of the modeled currents resembles the observations (Fig. 3), their amplitude and vertical extent are systematically smaller than in the observations (Figs. 1, 3); in the Atlantic, only a hint of the currents appears. Furthermore, although in the Atlantic the EICS is found in both models west of about $20^{\circ} \mathrm{W}$ in accord with the observations (Fig. 3), in the Pacific it is found across almost the entire basin in the I98 model, as in the observations, but only west of the Gilbert Islands, near $175^{\circ} \mathrm{E}$, in OFES. The confinement of the currents to the western Pacific in OFES puts into question their relevance to the observed EICS.

To our knowledge, no theory involving direct forcing by the mean wind can explain the EICS. Recently, d'Orgeville et al. (2007, hereafter D07) and Hua et al. (2008, hereafter H08) used a highly resolved OGCM to suggest that both the Atlantic EDJs and EICS result from the instability of an intraseasonal (about 60-day period) Yanai (Rossby gravity) wave. The wave was forced at the western boundary of the basin and had the vertical structure of a second baroclinic mode. When it had a sufficiently small zonal wavelength (about $3^{\circ}$ ), it became unstable, resulting in small-vertical-scale motions resembling the EDJs and large-vertical-scale motions resembling the EICS. A limitation of the solution, however, is that the model EICS is confined within a few degrees from the western boundary, inconsistent with the observations. To address this problem, Ménesguen et al. (2009; hereafter M09) extended the D07 and H08 studies by using a forcing confined to the upper $2500 \mathrm{~m}$ that excited baroclinic Yanai waves and barotropic short Rossby waves. The former generated the EDJs and the latter generated the EICS; in this simulation, the barotropic waves propagated fast enough to extend tens of degrees eastward before becoming unstable, thereby extending the zonal extent of the EICS. One limitation of these studies is the artificial nature of the forcing, for which there is no clear observational basis. Another limitation is that they do not address the quasi-steady dynamical balance that maintains the EICS.

In this paper, we propose an alternative way to generate the EICS using a wave forcing motivated by observations and OGCMs, and we address the question of the dynamical balance. The key mechanism that generates and maintains the EICS is self-advection combined with dissipation of the wave field, typically by wave breaking. Specifically, in the absence of closed PV contours, Lagrangian-mean circulation requires that water parcels change their potential vorticity (PV). Away from the surface, PV can change irreversibly only via dissipative processes. In the interior, away from the western boundary, a dissipating eddy or wave field can provide the necessary PV change to close the circulation in that area.

The preceding paragraph is linked to a main result of the wave-mean-flow interaction theory, the so-called nonacceleration theorem. Originally developed for the atmosphere, the theorem states that a zonally averaged flow can be generated by waves only if the waves are breaking, a process that involves, in particular, wave dissipation (Andrews and McIntyre 1976; Boyd 1976; Dunkerton 1980; McIntyre and Palmer 1983, 1984, 1985; McIntyre and Norton 1990). A variation of the theorem 
(a)
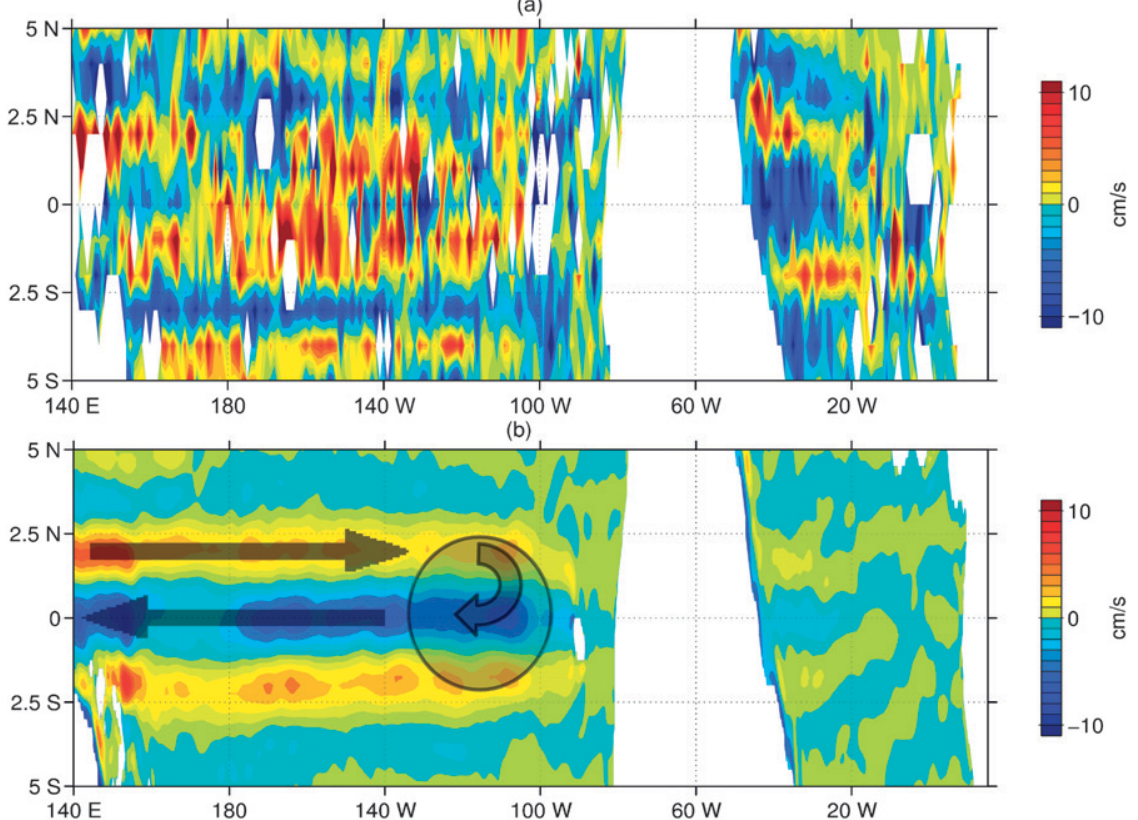

(c)

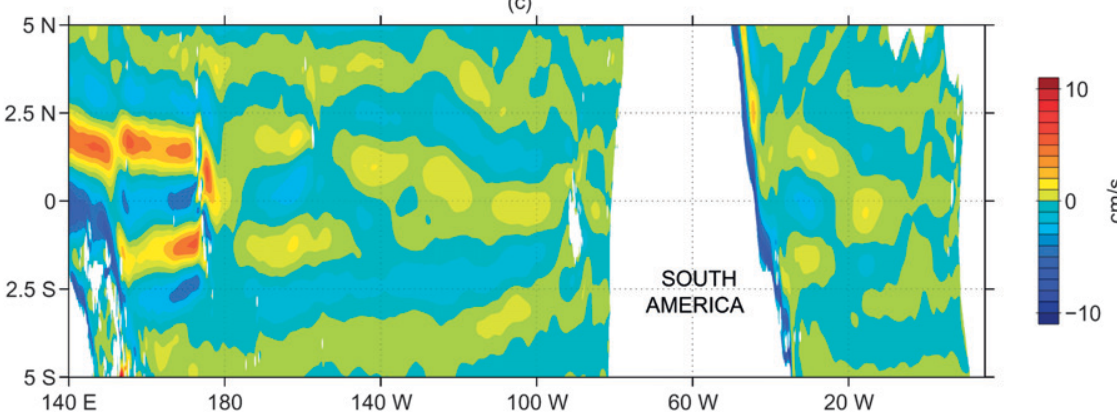

FIG. 3. Mean $u$ from (a) Argo floats at $1000 \mathrm{~m}$ and (b) the 198 model and (c) OFES averaged between 500 and $1000 \mathrm{~m}$. Time means are the same as in Figs. 1 and 2. An illustration of the mechanism suggested to trigger the EICS currents is superimposed in (b): the currents are part of a circulation (arrows) that is generated westward from and recirculates within a beam of dissipated Yanai waves in the eastern basin (shaded disk).

relevant to oceanography applies to time-mean flows, in which case, the theorem states that no steady Lagrangian flow in a rotating basin with no closed PV contours is possible if the waves are conservative (Moore 1970; see also section 4). A corollary is that, if there is no dissipation, the Eulerian-mean flow is exactly cancelled by the wave-induced Stokes drift, so the net displacements of water parcels are zero over a wave cycle. Hence, we use the decomposition of the Lagrangian-mean flow $\mathbf{U}_{L}$ into the Eulerian-mean flow $\mathbf{U}_{E}$ and the Stokes $\operatorname{drift} \mathbf{U}_{S}$

$$
\mathbf{U}_{L}=\mathbf{U}_{E}+\mathbf{U}_{S},
$$

to show explicitly the role of dissipation in controlling the Lagrangian circulation. The Eulerian-mean flow, which is often the quantity measured, is then obtained by subtracting the Stokes drift from the Lagrangian-mean flow.

In this study, we use idealized numerical and analytical ocean models to study the response forced by a beam of monthly periodic Yanai waves, which is generated near the surface by instabilities of surface currents and then radiates into the deep ocean. The beam is restricted in longitude and decays as it descends eastward, changing the PV of water parcels and hence enabling the generation of mean, equatorward flows on either side of the equator (Fig. 3b). These flows, in turn, generate mean zonal flows (the model EICS), which extend westward from the beam to the western boundary via the propagation of long Rossby waves during spinup. There, the PV changes are reversed in viscid, western boundary currents, where the water parcels return to their original latitudes. 
The paper is organized as follows: Section 2 reviews evidence for monthly periodic Yanai beams in both observations and models of the Pacific and Atlantic Oceans. Model results from previous works as well as from a new analysis of the I98 and OFES models are reported. Section 3 describes our numerical model and then reports solutions in which a beam of Yanai waves generates mean flows that resemble the EICS; the dissipation of the Yanai beam in these simulations is shown to result from its breaking via a complex cascade of energy toward the model grid scale. Section 4 explains the dynamics by presenting an approximate analytical solution for the EICS as a result of a Yanai beam dissipated by Rayleigh friction and compares it to the numerical solutions. To conclude, section 5 discusses the strengths and weaknesses of the theory and its relation to other theories.

\section{Monthly periodic Yanai waves}

Intraseasonal Yanai waves are hypothesized to modify water parcel PV so as to drive the EICS. These waves are found in the central-eastern Pacific and western-central Atlantic, where they form beams propagating downward to the east. Here, we review their characteristics in observations and numerical models, first in the literature (section 2a) and then in our new analyses of the I98 and OFES solutions (section $2 b$ ). Because self-advection and dissipation of the waves play an important role in the formation of the EICS, we also discuss in section $2 b$ the amplitude, level of nonlinearity, and dissipation of the Yanai beams in the I98 and OFES models.

\section{a. Previous results}

\section{1) OBSERVATiOns}

Observations of deep intraseasonal variability near the equator are sparse. They are limited to about a halfdozen moored measurements of velocity in the Pacific and Atlantic Oceans.

In the eastern Pacific, Harvey and Patzert (1976) reported an intraseasonal motion near the ocean bottom near $95^{\circ} \mathrm{W}$. Although their time series was only two months long, they identified an oscillation with a 25-day period, $1000-\mathrm{km}$ zonal wavelength, and amplitude of $4 \mathrm{~cm} \mathrm{~s}^{-1}$, with phase propagating westward at $50 \mathrm{~cm} \mathrm{~s}^{-1}$. They interpreted it as a first-meridional-mode equatorial Rossby wave, but Cox (1980) later suggested that their data are also consistent with the signal being a Yanai wave. Farther west, Eriksen (1985) and Eriksen and Richman (1988; Fig. 4a) analyzed 2-yr time series near $145^{\circ} \mathrm{W}$ at depths of 1500 and $3000 \mathrm{~m}$, finding that energy was distributed over a broad band in frequency but a narrow band in zonal wavenumber. Although their error bars are large, they estimated that the motion was consistent with first-meridional-mode Rossby waves for periods of 45 days and longer and with Yanai waves for periods of 30 days and shorter, both with a zonal wavelength longer than $1000 \mathrm{~km}$. They suggested that both waves originated from instabilities of the upper equatorial currents in the central-eastern Pacific, particularly from tropical instability waves (TIWs). Observations of intraseasonal meridional motions at depth in the eastern Pacific near $110^{\circ} \mathrm{W}$ were also made as part of the Equatorial Pacific Ocean Climate Studies (EPOCS) program but to our knowledge were never reported in the literature. ${ }^{1}$ The spectrum, presented in Fig. 4b, shows a peak near the monthly period.

In the eastern Atlantic near $3^{\circ} \mathrm{W}$, Weisberg et al. (1979) and Weisberg and Horigan (1981; Fig. 4c) reported a monthly periodic Yanai wave between 500 and $2000 \mathrm{~m}$ in a 1.5-yr time series, with upward-propagating phase, zonal wavelength of about $1200 \mathrm{~km}$, and zonal phase speed of about $50 \mathrm{~cm} \mathrm{~s}^{-1}$. They inferred that the wave originated from the upper-ocean instabilities. Farther west, at $23^{\circ}$ and $10^{\circ} \mathrm{W}$, motions in the 20-45-day period band dominated the meridional velocity $v$, especially near 1500-m depth (Bunge et al. 2006, 2008; von Schuckmann et al. 2008).

\section{2) Models}

In numerical models of the tropical Pacific, Cox (1980) and Masina and Philander (1999) reported deep motions radiating from the upper-ocean instabilities, consistent with first-meridional-mode Rossby and Yanai waves. Cox (1980) fit the deep signal with 1.1-month period and $1000-\mathrm{km}$ zonal wavelength waves, with the Rossby waves being nearly barotropic and the Yanai waves having a vertical wavelength of about $1800 \mathrm{~m}$. In Masina and Philander (1999), the Rossby and Yanai waves were found to have zonal wavelengths of about 1200 and $900 \mathrm{~km}$, respectively.

In the Atlantic, Li and Chang (1999) described the full spectrum of equatorial waves present in their OGCM. Near 2000-m depth, they found the dominant signal west of $10^{\circ} \mathrm{W}$ to be a monthly periodic Yanai wave with a zonal wavelength of $800-1000 \mathrm{~km}$.

\section{b. 198 and OFES solutions}

\section{1) YANAI BEAM}

In the 198 and OFES solutions, monthly periodic Yanai waves form well-defined beams in both the central-eastern

\footnotetext{
${ }^{1}$ Tang et al. (1988) introduced the data, but only the motions with a period longer than 36 days were analyzed.
} 
(a) Central Pacific

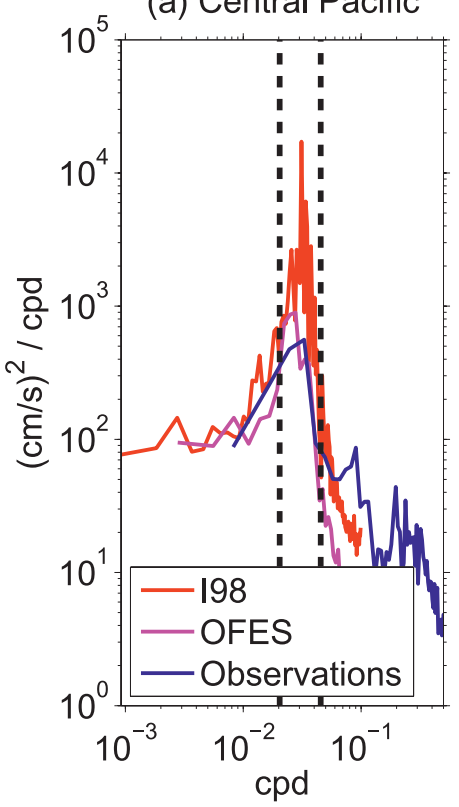

(b) Eastern Pacific

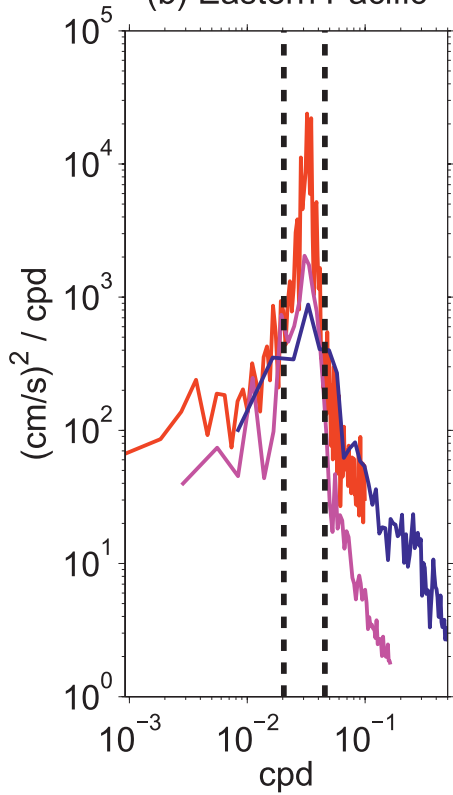

(c) Eastern Atlantic

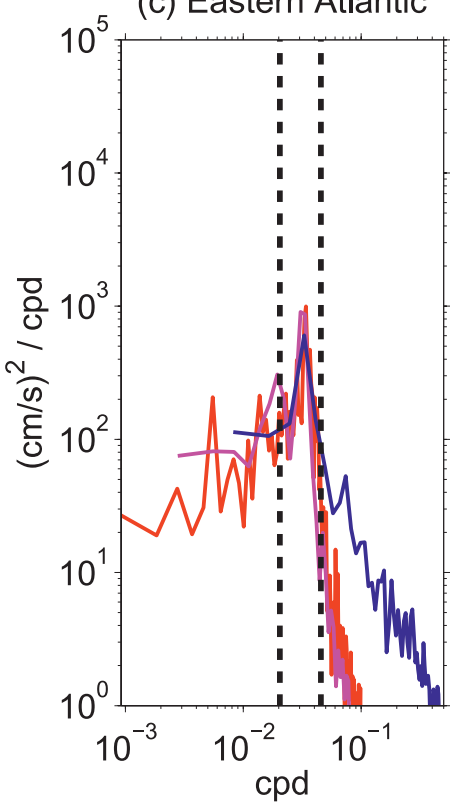

FIG. 4. Averaged power spectrum density (PSD) in equatorial $v$ from the 198 model (red) and OFES (magenta) compared to observed ones (blue), where cycles per day (cpd) are used as a unit. The locations of the moorings are shown in Fig. 5. The model PSD are obtained from years 20-22 in the I98 model and year 46 in OFES. They are averaged between 400 - and $1000-\mathrm{m}$ depth and over (a) $148^{\circ}-138^{\circ} \mathrm{W}$, (b) $111^{\circ}-110^{\circ} \mathrm{W}$, and (c) $4^{\circ}-3^{\circ} \mathrm{W}$. Observations are (a) near $145^{\circ} \mathrm{W}$ from the Pacific Equatorial Ocean Dynamics (PEQUOD) program during 1980-83 (Eriksen and Richman 1988), (b) near $110^{\circ} \mathrm{W}$ from EPOCS during 1980-82 (Tang et al. 1988), and (c) near $4^{\circ} \mathrm{W}$ from Weisberg and Horigan (1981) during 1977-78, with selected moorings between 400 and $1000 \mathrm{~m}$ and within $0.1^{\circ}$ from the equator. The 22-49-day-period band is limited by the dashed lines.

Pacific and western-central Atlantic (Figs. 4, 5). In the Pacific, the Yanai waves have a period of 30 days and a zonal wavelength of $1000 \mathrm{~km}$; in the Atlantic, a period of 35 days and a zonal wavelength of $750 \mathrm{~km}$. These are within the range of previous estimates from observations and models. The beams are modulated annually, as in the observations, because of the annual cycle of the upper-ocean instabilities that generate them (e.g., Menkes et al. 2002; Lyman et al. 2007). Because few observations are available, however, we do not know if the beams in these models have a realistic structure.

\section{2) Amplitude}

The amplitude of the monthly periodic Yanai waves in the observations and models is estimated by the depthtime mean amplitude of $v$ at the equator between 400 and $1000 \mathrm{~m}$ in the 22-49-day band obtained from complex demodulation of the time series at each depth. In the Atlantic, the amplitude observed east of the beam near $3^{\circ} \mathrm{W}$ is about $4 \mathrm{~cm} \mathrm{~s}^{-1}$, whereas the I98 and OFES models yield about $5 \mathrm{~cm} \mathrm{~s}^{-1}$. From $40^{\circ}-20^{\circ} \mathrm{W}$, the wave amplitude is about $9 \mathrm{~cm} \mathrm{~s}^{-1}$ in the two models, consistent with the observations at $23^{\circ} \mathrm{W}$ of Bunge et al. (2008). In the Pacific, the observed amplitude is about
$6 \mathrm{~cm} \mathrm{~s}^{-1}$ near $142^{\circ} \mathrm{W}$ and $7 \mathrm{~cm} \mathrm{~s}^{-1}$ near $110^{\circ} \mathrm{W}$, whereas the corresponding amplitudes are 1-1.3 times larger in OFES and 2-3 times larger in the I98 model. The high amplitude in the I98 model presumably results from the unrealistically strong equatorial wind field used to force it (Harrison 1989). On the other hand, the observations may be biased low by the weaker than normal upperocean instabilities and waves caused by the 1982/83 El Niño being included in the periods of observation (Yu and Liu 2003). Hence, the OFES Yanai wave amplitude may be realistic as a long-term mean.

\section{3) NONLINEARITY}

Two characteristics of the Yanai waves are essential to the present theory: 1) finite amplitude and 2) dissipation. A measure of nonlinearity for waves is the ratio $M$ of the amplitude of the wave velocity in the direction of phase propagation to its phase velocity (Gill 1974). For a Yanai wave,

$$
M=\frac{U}{\left|\omega_{A} / k_{A}\right|}=\frac{\left|k_{A}\right| V}{\sqrt{\beta c_{A}}}
$$

where $\omega_{A}$ is the frequency of the Yanai wave; $k_{A}$ is its zonal wavenumber, $U$ and $V$ are the amplitude of $u$ and 
(a)

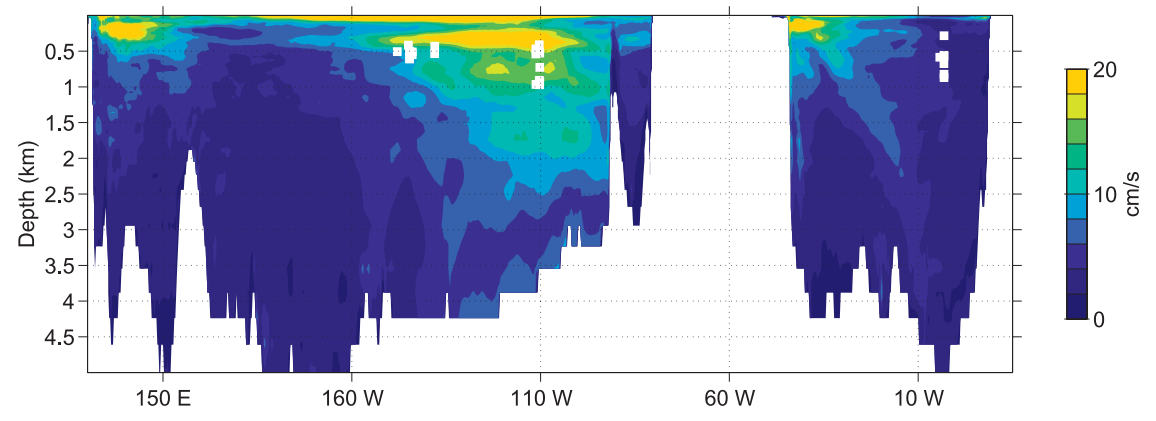

(b)

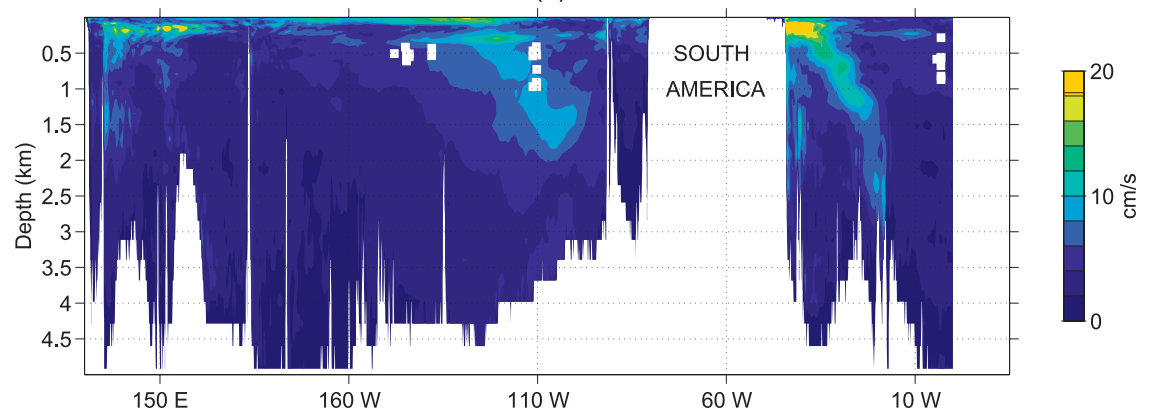

FIG. 5. Standard deviation (STD) of equatorial $v$ in (a) the I98 model during years 20-22 and

(b) OFES during year 46. White squares show the location of the moorings used in Fig. 4.

$v$, respectively; $\beta$ is the planetary vorticity gradient; and $c_{A}$ is the gravity wave speed. The second equality in (2) is obtained using the relative scaling of $u$ and $v$ for a Yanai wave (see appendix). For a 30-day and 1000-km Yanai wave, $c_{A}$ is about $53 \mathrm{~cm} \mathrm{~s}^{-1}$ and $M$ within the Pacific beam is about $0.1-0.15$ in the observations, $0.1-0.2$ in OFES, and 0.1-0.4 in the I98 model. Within the Atlantic beam, it is about $0.1-0.3$ in both models. The waves are thus weakly to moderately nonlinear.

\section{4) Dissipation}

Dissipation of the beam is needed to obtain the EICS. A discussion of the cause of that dissipation based on the present numerical simulations is given in section $3 \mathrm{~d}$. Here, we only note that the Yanai waves in the observations and models decay downward and eastward along the beam axis. As in the analytical solutions (section 4), the effective dissipation is parameterized by Rayleigh friction, with a coefficient $r$ that can be calculated approximately from the vertical decay of the Yanai beam. After removing the effect of the depth-variable stratification via stretching and scaling (Gill 1982), the decay is computed from the profile of the Yanai wave amplitude. One obtains $r / \omega_{A}$ of about $0.01-0.03$ for the beam in the Pacific and Atlantic in the I98 model; with a time scale of 6-18 months, the dissipation is weak.

\section{Numerical solutions}

In this section, numerical simulations are used to test the hypothesis that an intraseasonal Yanai beam can generate the EICS. We first introduce the model, its configuration and the set of experiments (section 3a). We then present results from two solutions, which illustrate the importance of dissipation to obtain an EICS that extends across the basin: one with weak forcing and negligible beam decay and the other with standard forcing and substantial beam decay (section $3 \mathrm{~b}$ ). The sensitivity of the solution to the amplitude of the forcing is studied further with additional experiments (section 3c). We conclude by discussing the breaking of the Yanai beam and the cascade of energy toward small scales responsible for the beam decay (section 3d).

\section{a. Model configuration and experiments}

In this section, idealized numerical simulations are performed to study the formation of the EICS from a dissipated beam of monthly periodic Yanai waves. The Parallel Ocean Program (POP) model (e.g., Maltrud and McClean 2005) is used. In all experiments, it is configured with 100 levels uniformly spaced over the 5000-m water column for a vertical grid spacing of $\Delta z=$ $50 \mathrm{~m}$. The horizontal grid spacing $\Delta x$ is $1 / 4^{\circ}$ in both longitude and latitude as in the $\mathrm{I} 98$ model. 
(a)

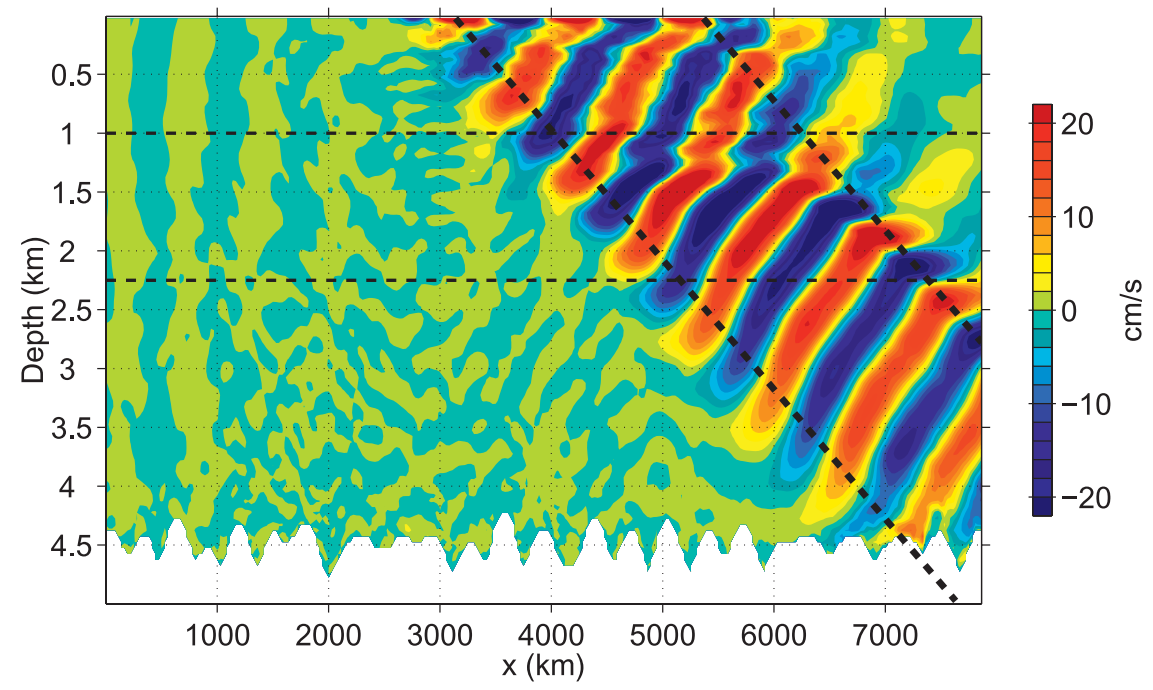

(b)

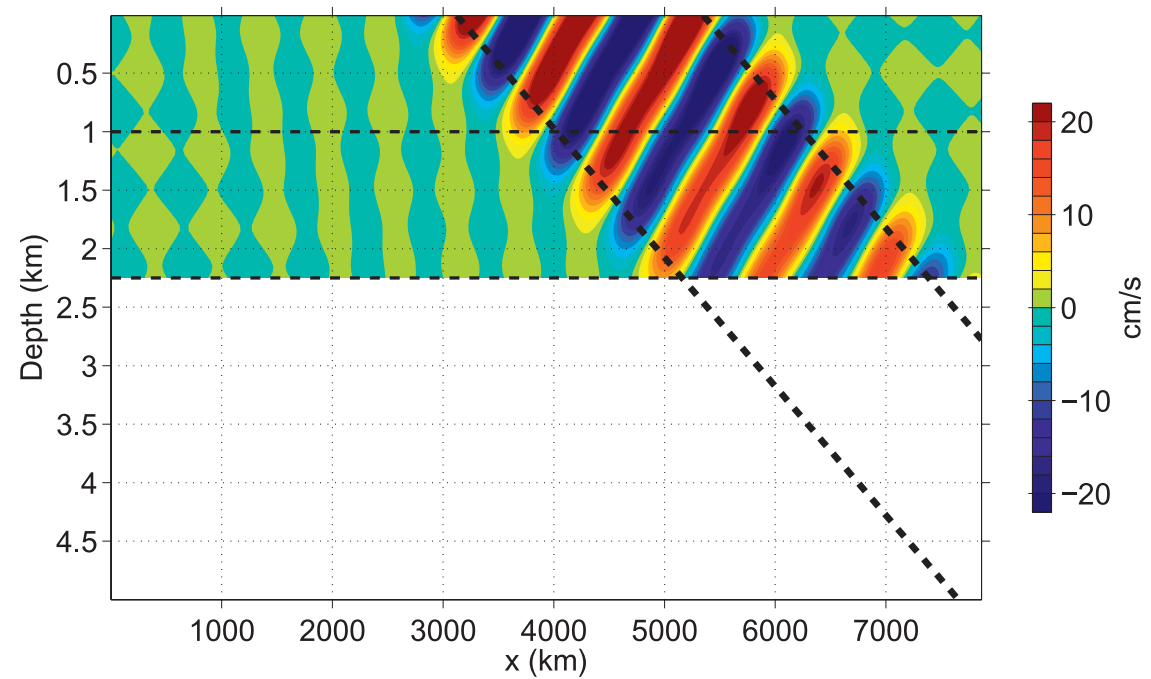

FIG. 6. Snapshot of $v$ along the equator in the standard case: (a) numerical solution (N0.5) and (b) analytical solution. The slanted dashed lines show the ray slope expected by theory and the edges of the beam within which zonal averages are taken. The horizontal lines show the depth range (from $z_{\max }=-1000 \mathrm{~m}$ to $z_{\min }=-2250 \mathrm{~m}$ ) over which averages along the beam are performed.

The basin is rectangular, extending from $20^{\circ} \mathrm{S}$ to $20^{\circ} \mathrm{N}$ and $72^{\circ}$ in longitude. It has vertical walls and an irregular bottom topography, designed to reduce the reflection of inertia-gravity waves generated by the self-advection of the Yanai beam. Bottom friction is included using a quadratic bottom drag formula with a dimensionless coefficient of $2 \times 10^{-3}$. Biharmonic horizontal mixing is used with coefficients for momentum and tracers of $-2 \times 10^{14} \mathrm{~m}^{4} \mathrm{~s}^{-1}$. The vertical mixing scheme of Pacanowski and Philander (1981) is adopted with a background diffusivity of $10^{-5} \mathrm{~m}^{2} \mathrm{~s}^{-1}$ for both momentum and tracers.
Each simulation starts with the ocean at rest and a horizontally uniform linear profile of background potential density $\hat{\rho}(z)=\hat{\rho}_{z} z$. The background buoyancy frequency is $\hat{N}=\sqrt{-g \hat{\rho}_{z} / \rho_{0}}=2 \times 10^{-3} \mathrm{~s}^{-1}$, where $g$ is the gravitational acceleration and $\rho_{0}$ is a constant potential density. With temperature restored at the surface on a time scale of a month, salinity uniform and constant at 35 psu, and weak tracer diffusion, the instantaneous stratification deviates little from the background stratification. All time averages are performed in isopycnal coordinates for consistency with the analytical development in section 4. For plotting, $\rho$ is mapped back to $z$ using the background field. 
TABLE 1. List of the six experiments with their name, the STD $\tau$ of the surface stress, and their type. The number appearing in each experiment name equals the $\tau$ value used. All experiments are run with 100 levels and $1 / 4^{\circ}$ resolution in the horizontal. Properties that differ from their standard value in N0.5 are shown in bold.

\begin{tabular}{lll}
\hline \hline Name & $\tau\left(\right.$ dyn cm $\left.^{-2}\right)$ & Type \\
\hline N0.5 & $\tau_{0}(0.5)$ & NB \\
N0.05 & $\tau_{0} / \mathbf{1 0}(\mathbf{0 . 0 5})$ & NB \\
N0.125 & $\tau_{0} / \mathbf{4}(\mathbf{0 . 1 2 5})$ & NB \\
N0.25 & $\tau_{0} / \mathbf{2}(\mathbf{0 . 2 5})$ & NB \\
N1 & $\mathbf{2} \tau_{0}(\mathbf{1})$ & NB \\
N0.5_BB & $\tau_{0}(0.5)$ & BB \\
\hline
\end{tabular}

All simulations are run for $10 \mathrm{yr}$, and annual or monthly averages are archived for the entire run. The initial transients are evident only within the first $3 \mathrm{yr}$. Analyses are performed during the last $3 \mathrm{yr}$ of the runs (years 7-9), for which 5-day averages (called "snapshots") have been archived. One experiment (N0.25) was run for an additional $11 \mathrm{yr}$ to explore low-frequency variability and the cause of beam decay (section 3d).

For simplicity, we do not force the model with realistic winds that can generate unstable surface currents. Instead, to mimic the generation of a beam of monthly Yanai waves by upper-ocean instabilities, we force the ocean with a patch of meridional wind stress located in the center of the basin:

$$
\tau^{Y}=\tau X(x) Y(y) \sin \left(k_{A} x-\omega_{A} t\right)
$$

To form a beam, the zonal profile $X(x)$ extends only $20^{\circ}$ in longitude with a $5^{\circ}$ taper to zero on each side. The meridional profile $Y(y)$ is a $6^{\circ}$-wide Blackman window centered on the equator.

With one exception, the spectrum of the surface stress is narrow band (NB) in both frequency and zonal wavenumber, with $\omega_{A}=2 \pi / 33$ day $^{-1}$ and $k_{A}=-2 \pi / 1000 \mathrm{~km}^{-1}$. The forcing is thus a westward-propagating wind that excites a Yanai wave with a vertical wavelength of about $1700 \mathrm{~m}$ and a gravity wave speed $c_{A}$ of about $53 \mathrm{~cm} \mathrm{~s}^{-1}$. The exception is one experiment (N0.5_BB) where the spectrum of the forcing is broad band (BB), to mimic the modulation of the Yanai waves by the annual cycle and interannual variability. The BB forcing is the sum of randomly phased waves with periods varying from 27 to 43 days and zonal wavelengths varying from 770 to $1430 \mathrm{~km}$, consistent with observations (section 2).

Figure 6a shows a snapshot of $v$ at the equator in one typical experiment (N0.5): the forcing excites a beam that propagates downward and to the east, consistent with ray theory. The beam reaches the 5000 -m ocean bottom within a year. It then ultimately reaches the eastern boundary, either directly or after reflection at the bottom into an eastward and upward beam. At the eastern boundary, the

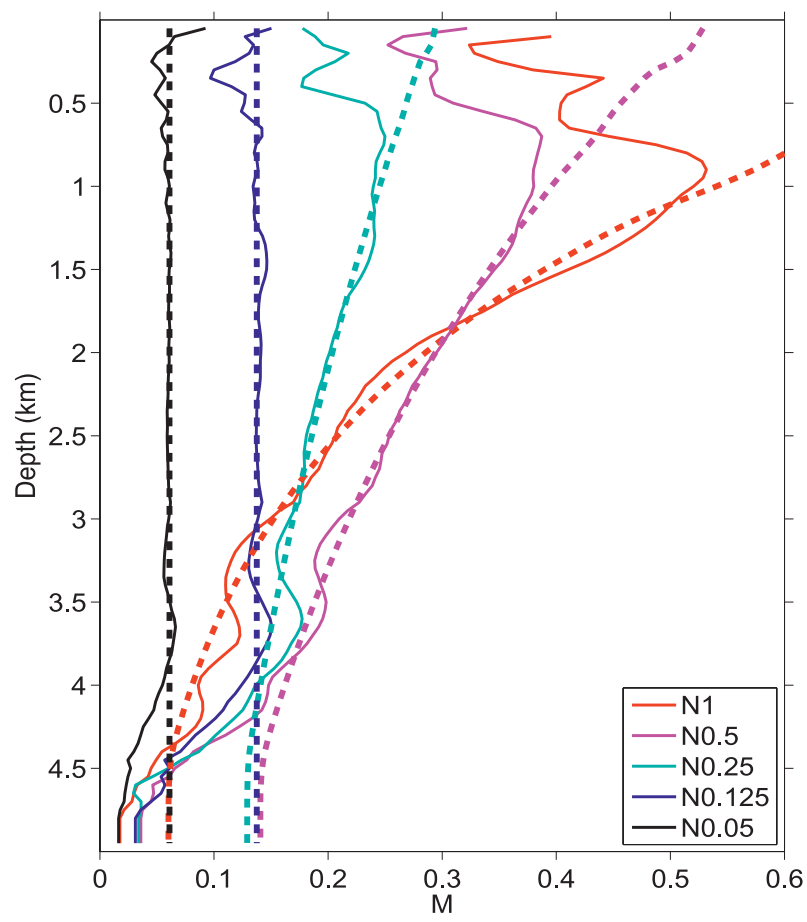

FIG. 7. Vertical profiles of the beam amplitude in the runs with varying forcing amplitude constructed from the amplitude of equatorial $v$ at the 1.1-month period and averaged between the two edges of the beam shown in Fig. 6. The amplitude is plotted as the measure $M$ of wave nonlinearity, $M=|k| V / \sqrt{\beta c_{A}}$, where $k=k_{A}=$ $-2 \pi / 1000 \mathrm{~km}^{-1}, c_{A} \approx 53 \mathrm{~cm} \mathrm{~s}^{-1}$, and $V$ is the amplitude of the meridional velocity. The dashed lines are exponential fits (see text).

beam reflects into coastal Kelvin waves (McCreary 1984) that propagate the energy poleward, where it is dissipated along the basin boundaries (Fig. 6a). In N0.5_BB, the resulting beam broadens only slightly with depth and is otherwise similar to the beam shown in Fig. 6a.

Six experiments that differ in their surface stress have been performed (Table 1). Experiment N0.5 is central; the stress has a "standard" amplitude of $\tau=\tau_{0}=0.5 \mathrm{dyn}$ $\mathrm{cm}^{-2}$ and forces a beam with an amplitude of about 15$20 \mathrm{~cm} \mathrm{~s}^{-1}$ between 500- and 1500-m depth, similar to that in the Pacific in the 198 model. Experiments N0.05, $\mathrm{N} 0.125, \mathrm{~N} 0.25$, and $\mathrm{N} 1$ explore the sensitivity of the numerical solution to the amplitude of the forcing. They are forced with weak $\left(\tau_{0} / 10\right.$ and $\left.\tau_{0} / 4\right)$, moderate $\left(\tau_{0} / 2\right)$, and strong $\left(2 \tau_{0}\right)$ forcings, respectively. Experiment N0.5_BB is identical to N0.5 except that the forcing is BB.

\section{b. Weak and standard forcings: N0.05 and N0.5}

Results from the weak (N0.05) and standard (N0.5) experiments are described in parallel to illustrate the key role of dissipation. Although the amplitude of the Yanai beam is relatively depth independent in the first case, it decays in the second (Fig. 7). The decay is due to 
(a)

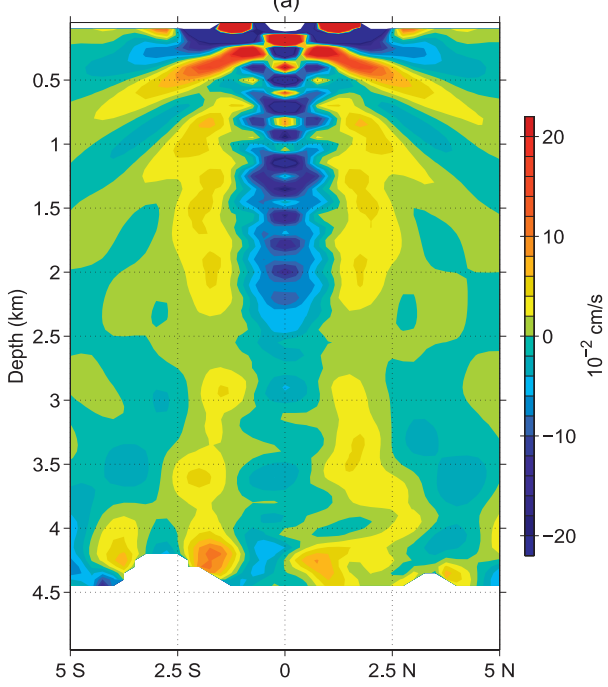

(c)

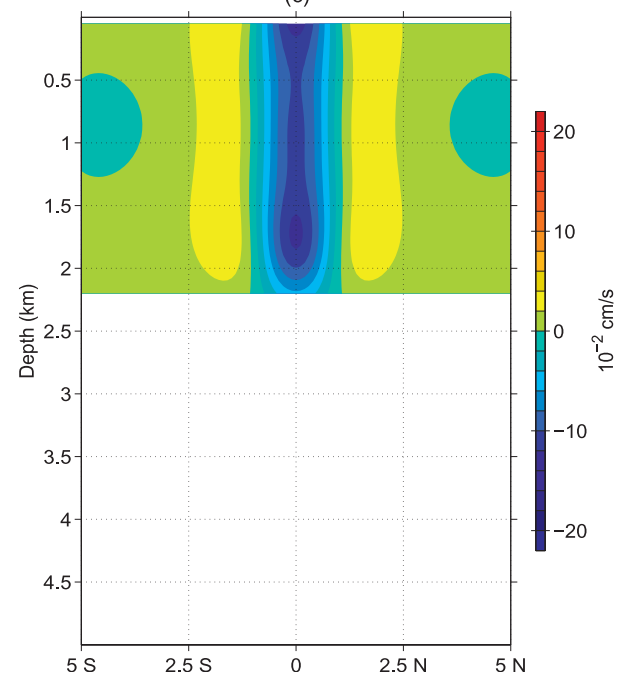

(b)

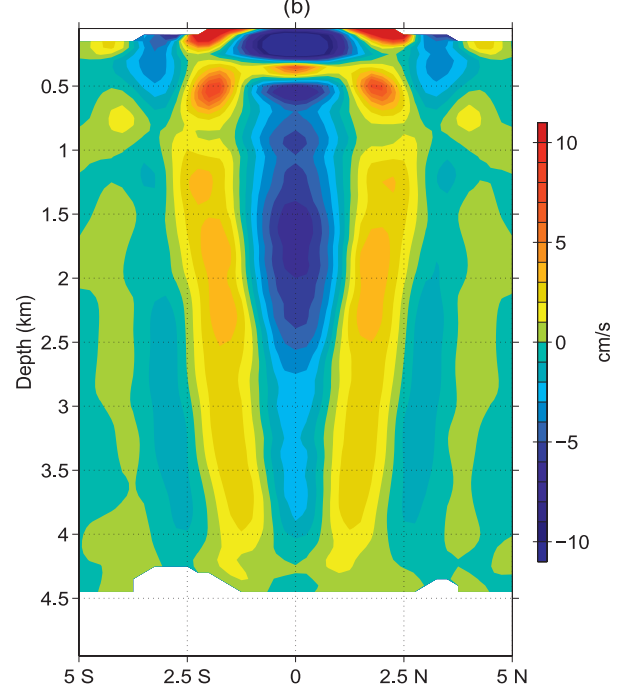

(c)

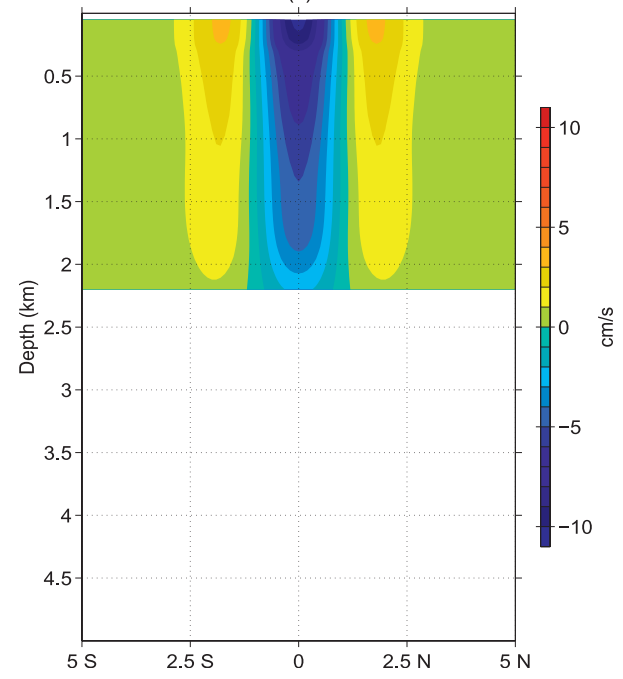

FIG. 8. Mean Eulerian zonal velocity $U_{E}$ at $x=5000 \mathrm{~km}$ over model years $7-9$ from the (a) weak (N0.05) and (b) standard (N0.5) numerical experiments and over a wave cycle from the (c) weak and (d) standard analytical solutions. Here and in all subsequent figures, time averaging is performed along isopycnals and plotted against $z$ (see section 3.1).

the breaking of the Yanai beam, which drains its energy down to the model's grid scale where it is dissipated. This cascade of energy is discussed further in section $3 \mathrm{~d}$, but for now we simply note that the beam can be considered inviscid in the weak case, whereas it is effectively dissipated with depth in the standard case.

In both cases, within the beam and below $1000 \mathrm{~m}$, there appears a set of large-vertical-scale Eulerian-mean zonal currents forming a structure resembling the EICS within $2.5^{\circ}$ from the equator: a westward current on the equator and eastward currents near $2^{\circ}$ from the equator as seen in Figs. 8a,b, (and Fig. 9). In the standard case, an additional pair of westward currents is found near $3^{\circ}$ from the equator, consistent with the EICS. The velocity extrema are similar to those obtained in the Pacific in the I98 model, but they are weaker than in the observations of Firing (1987), especially with respect to the eastward current at $2^{\circ} \mathrm{S}$.

Apart from amplitude, the largest difference between the two experiments is the zonal extent of the currents as shown in Figs. 10a,b. In the weak case, the currents are constrained to lie within the beam, whereas in the standard case they extend westward to the boundary, with the same large-vertical-scale structure as within the beam. This difference, together with the difference in the vertical decay of the beam, suggests that dissipation of the Yanai waves is the key to obtaining currents over a large portion of the basin. 


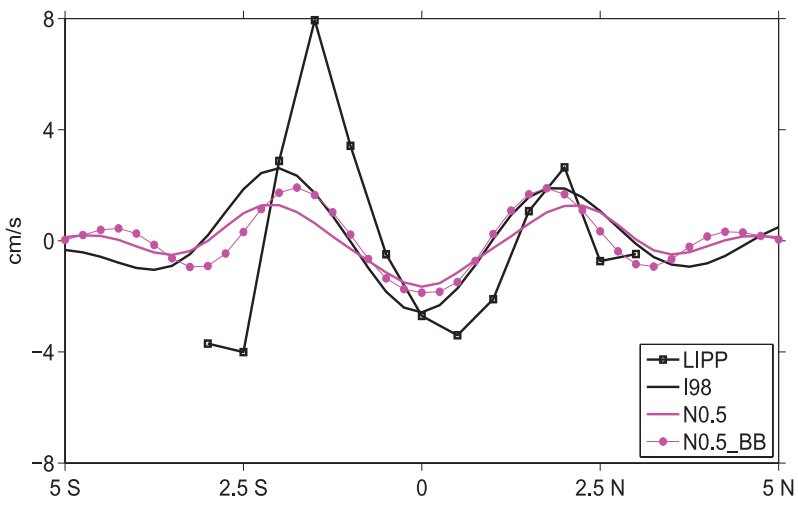

FIG. 9. Mean Eulerian zonal velocity $U_{E}$ at $159^{\circ} \mathrm{W}$ averaged between 400 and $1500 \mathrm{~m}$ from LIPP observations and years 20-22 of the 198 model, and west of the beam (at $x=3000 \mathrm{~km}$ ) and alongbeam averaged between $z_{\max }$ and $z_{\min }$ (see Fig. 6) over years $7-9$ of N0.5 and N0.5_BB.

\section{c. Sensitivity to forcing}

To quantify how the amplitude and the decay scale vary with the forcing amplitude, each Yanai beam profile of Fig. 7 is fit by an exponentially decaying profile between 1000- and 3000-m depth:

$$
V(\rho) \approx c_{A} \epsilon_{A}(\rho)=c_{A} \epsilon_{A, 0} e^{-m_{A}^{i}\left(\rho / \hat{\rho}_{z}\right)},
$$

where $\epsilon_{A, 0}$ is the dimensionless amplitude of the exponential profile at $\rho=0(z=0)$ and $m_{A}^{i}$ is its decay rate (unit of $\mathrm{m}^{-1}$ ) with depth. The resulting profiles are plotted as dashed curves in Fig. 7. As we shall see in section 4, these profiles correspond approximately to the analytical solution for a Yanai beam dissipated by Rayleigh friction, for which $m_{A}^{i}$ is the imaginary part of the vertical wavenumber $m_{A}$ and is related to the Rayleigh friction coefficient $r$. In the present regime of weak dissipation $\left(r / \omega_{A} \ll 1\right), m_{A}^{i}$ is nearly proportional to $r$.

As expected, the amplitude of the Yanai beam itself varies linearly with the amplitude of the forcing (not shown). Except in the two weak experiments (N0.05 and N0.125) where they are virtually zero, the decay rate $m_{A}^{i}$ and the effective dissipation $r$ also increase with the forcing amplitude (N0.25, N0.5, and N1; Fig. 7); hence, they also increase with the beam amplitude (Fig. 11, black line). This relationship is consistent with the idea that the dissipation arises from the cascade of energy toward the model's grid scale via the breaking of the Yanai waves (see section 3d). The fact that there is virtually no dissipation in the two weak experiments suggests that either 1) the cascade of energy has not yet developed in these two experiments or 2) there is a threshold in amplitude below which the cascade does not occur.

As in the weak and standard cases, the EICS currents within $2.5^{\circ}$ from the equator are obtained within the beam in all experiments and to the west of the beam only when the beam is dissipated with depth. The amplitude of the EICS currents within the beam varies quasi quadratically with the beam amplitude (Fig. 11, red and blue lines), suggesting that the EICS is generated by the selfadvection of the beam. For the strongest beam, however, the EICS currents are weaker than expected from

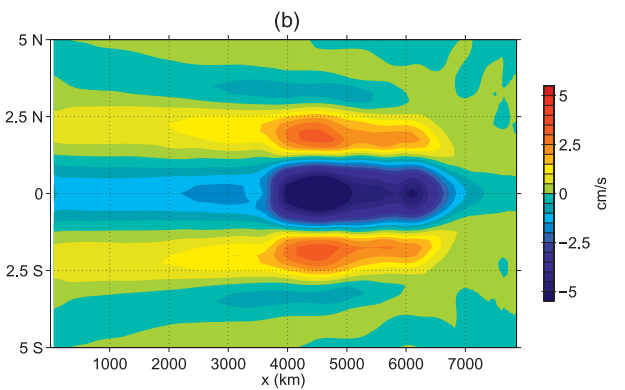

(d)

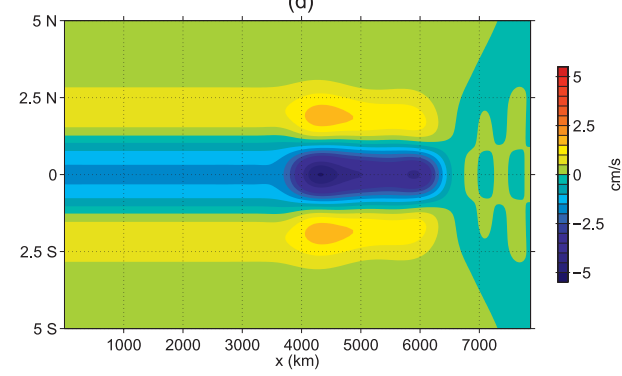

FIG. 10. As in Fig. 8, but for top view of the mean Eulerian zonal velocity. The flow is averaged along the beam between $z_{\max }$ and $z_{\min }$ (see Fig. 6) and plotted vs $x$ of $z_{\max }$. 


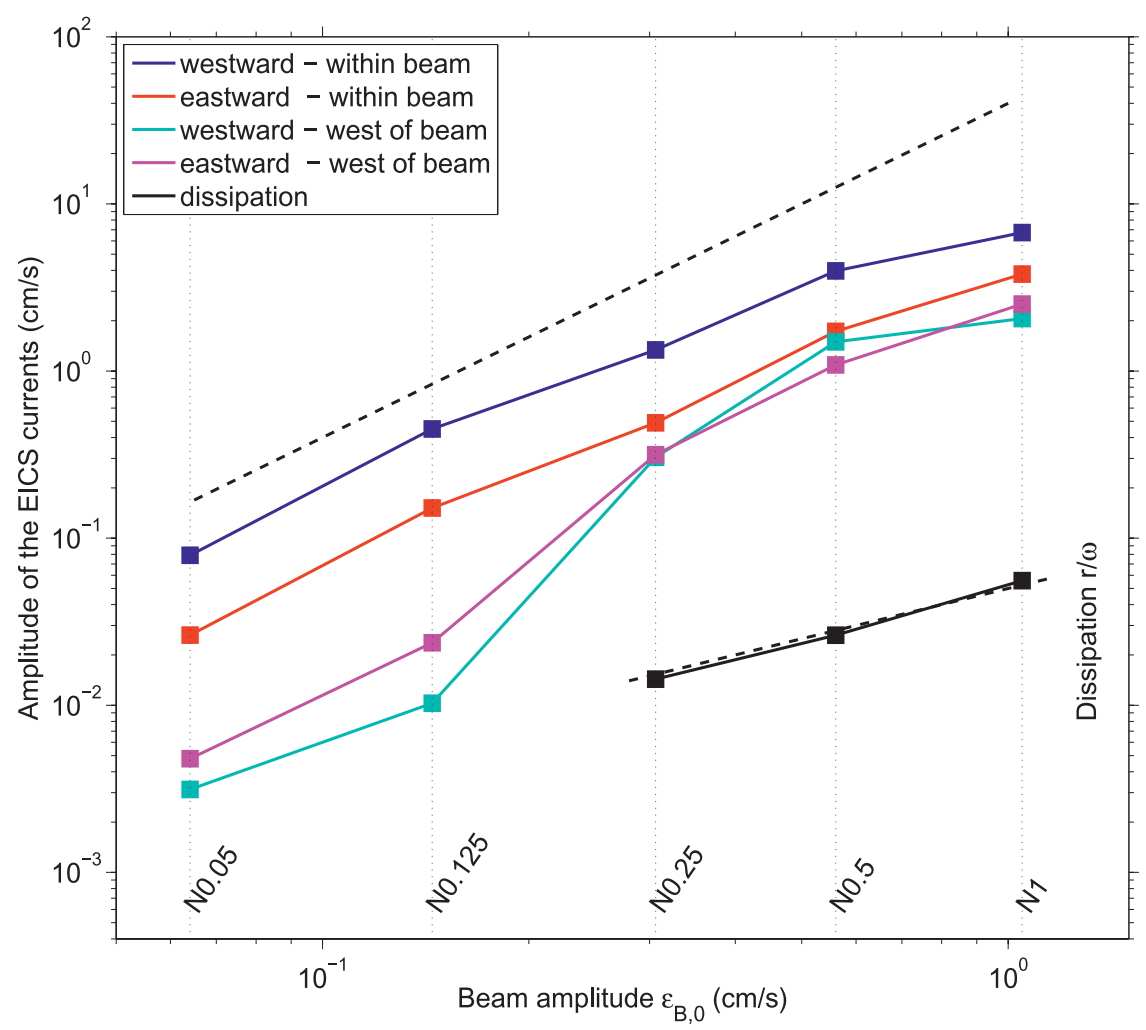

FIG. 11. Amplitude ( $\mathrm{cm} \mathrm{s}^{-1}$; colors) of the EICS currents and dimensionless dissipation $\left(r / \omega_{A}\right.$; black) vs the beam amplitude $\epsilon_{A, 0}\left(\mathrm{~cm} \mathrm{~s}^{-1}\right)$ at $z=0$ in the experiments varying with forcing amplitude. The amplitude is defined as the maximum speed of the current averaged as in Fig. 10 and subsequently averaged between $x=1000$ and $3000 \mathrm{~km}$ for the region to the west of the beam and between the two edges of the beam for the region within the beam: blue and cyan correspond to the westward current within $1^{\circ}$ from the equator and red and magenta correspond to the eastward current north of $1^{\circ} \mathrm{N}$. The dashed lines show a linear and quadratic law.

a quadratic relationship. The analytical solution (section 4) suggests that this discrepancy arises because the dissipation is also increasing with the beam amplitude; with constant dissipation, the relation is exactly quadratic.

The EICS currents west of the beam also strengthen with forcing amplitude, and the relation is quadratic as long as the beam is dissipated (Fig. 11, magenta and cyan lines). Because there is virtually no dissipation for the cases with weak forcing (N0.05 and N0.125), we do not expect the nonzero mean currents obtained west of the beam in these experiments to correspond to EICS currents; indeed, once the difference in beam amplitude is corrected, these currents are much weaker and have a different meridional structure than in the cases with dissipation (Fig. 12a). These currents are likely the result of nonlinear processes involving other high-frequency variability that may be a residual of the spinup or of the beam energy that has not been entirely dissipated along the basin boundaries.

Because the dissipation and the EICS both increase with the forcing, it is not clear how the EICS relates to the dissipation independently of the forcing. Based on results from our analytical model (section 4), we have found that a useful measure is to compare the amplitude ratio of the flows (west of/within) the beam against dissipation, a plot of which is shown in Fig. 13. The ratio is roughly constant provided the beam is dissipated; the westward equatorial current and eastward currents at $2^{\circ} \mathrm{N}-2^{\circ} \mathrm{S}$ to the west of the beam are about $20 \%-40 \%$ and $60 \%-80 \%$ weaker than those within the beam, respectively. This property suggests that, as long as dissipation occurs, the amplitude of the currents is independent of the level of dissipation. We will see in section 4 that this result is one inconsistency between the numerical and analytical solutions.

The meridional structure of the EICS within the beam is insensitive to the forcing amplitude (Fig. 12b); the main qualitative difference is that off-equatorial westward currents appear only in the experiments with standard and strong forcing (N0.5 and N1). West of the beam, however, the meridional structure of the EICS differs much more among the experiments (Fig. 12a). 

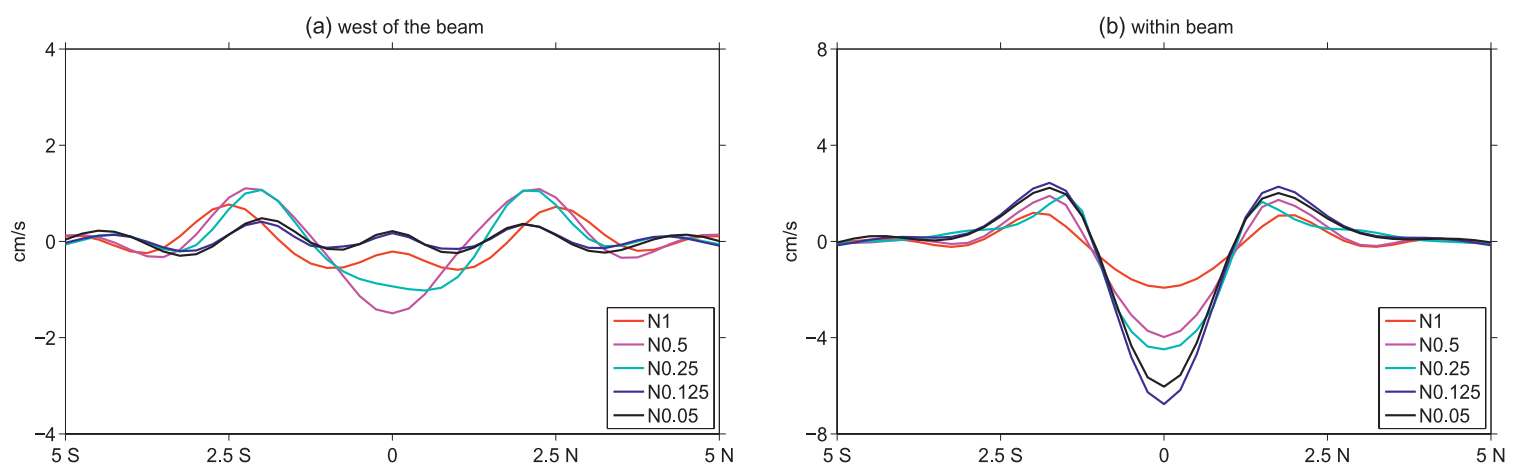

FIG. 12. Mean Eulerian zonal velocity $U_{E}$ in the numerical experiments varying in forcing amplitude. The velocity is along-beam averaged between $z_{\max }$ and $z_{\min }$ (see Fig. 6) over model years 7-9: (a) west of the beam (zonally averaged between $x=1000$ and $3000 \mathrm{~km}$ ) and (b) within the beam (zonally averaged between the beam edges). The velocity has been normalized by $\left[\epsilon_{A, 0}(\mathrm{~N} 0.5) / \epsilon_{A, 0}(\mathrm{Ni})\right]^{2}$ where $\epsilon_{A, 0}(\mathrm{Ni})$ is $\epsilon_{A, 0}$ calculated for experiment Ni. If the amplitude of the currents would vary exactly as a quadratic law with the beam amplitude, all profiles would collapse onto one.

With moderate (N0.25), standard (N0.5), and strong (N1) forcing, the structure is similar to that within the beam.

The beam and the EICS obtained in the experiment with $\mathrm{BB}$ forcing are similar to those in the standard run (Fig. 9). The main difference is that the off-equatorial currents are a bit stronger and shifted equatorward by
$1 / 2^{\circ}$. Thus, the mechanism responsible for the EICS in the experiments with NB forcing is not sensitive to a realistic modulation of the forcing.

\section{d. Cause of the beam decay}

The increase of dissipation with beam amplitude is consistent with the nonlinear transfer of the Yanai wave

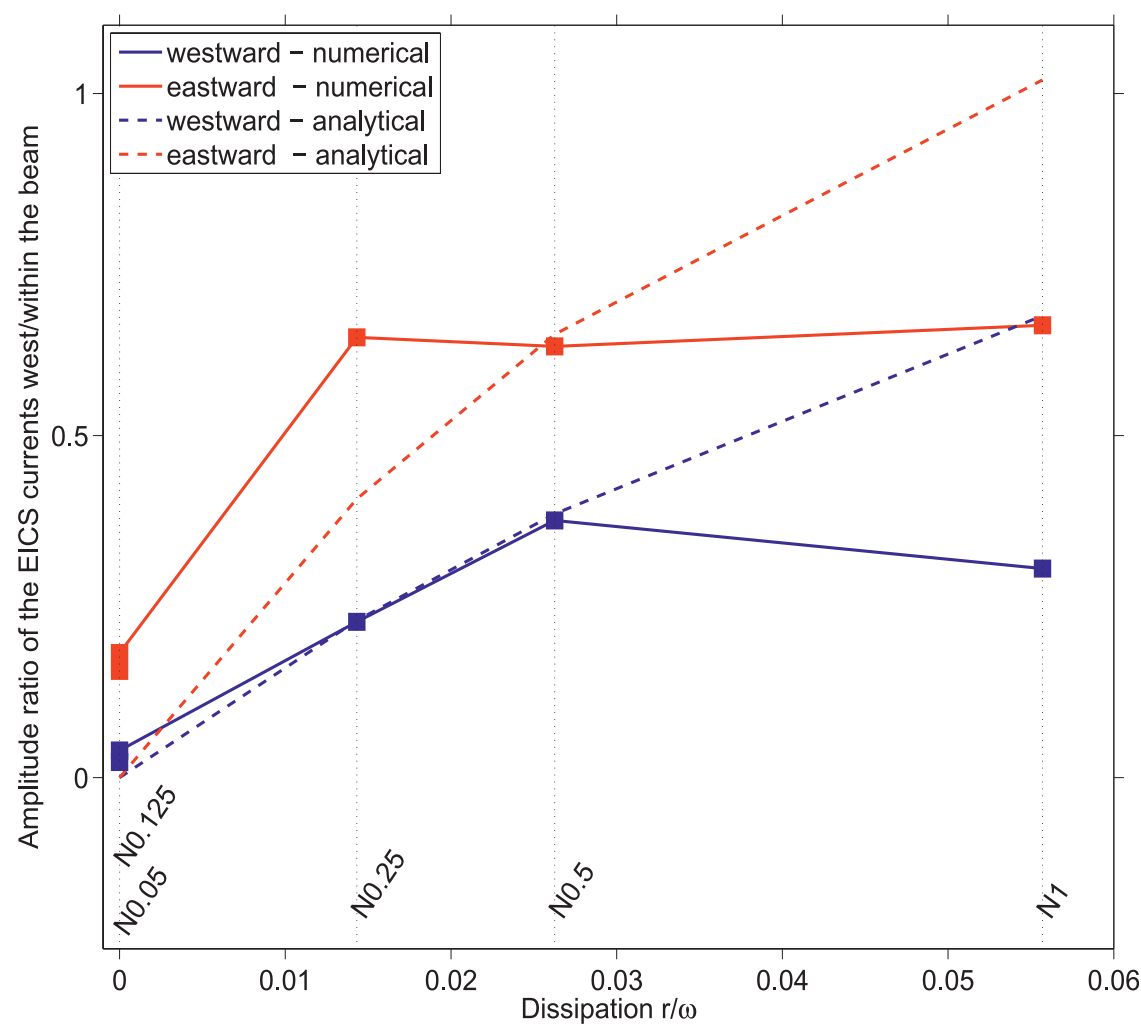

FIG. 13. Ratio of the amplitude of the EICs currents (west of/within) the beam vs $r / \omega_{A}$ : in the numerical experiments varying in forcing amplitude (solid lines) and the analytical solutions (dashed lines). The definition of the amplitude of the EICs currents is the same as in Fig. 11. 
energy to other frequencies and spatial scales. In the two weak experiments (N0.05 and N0.125), the beam is weakly nonlinear $(M \approx 0.05-0.15)$. Less than $2 \%$ of the total kinetic energy is found outside the forcing frequency band (32-34 days) and the profile of equatorial $V$ within the beam is sinusoidal (Fig. 14). In going from the moderate (N0.25) to standard (N0.5) to strong (N1) experiments, the beam becomes more and more nonlinear, with $M$ increasing from about 0.2 to 0.5 , energy outside the forcing frequency band rising from $17 \%$ to $30 \%$, and small-vertical-scale motions appearing superimposed on the Yanai beam (Fig. 14).

Although the energy is distributed continuously over frequency and wavenumber space in experiments with standard and strong forcing, it appears as a set of discrete peaks with moderate forcing (N0.25; Fig. 15), exposing the multiple nonlinear interactions responsible for the spread of energy over time and space. As described in section 4, the first energy transfer is from the directly forced Yanai wave (of frequency $\omega_{A}$ and wavenumber $k_{A} ; A$ in Fig. 15) to the mean flow (D in Fig. 15). The self-advection of the wave also transfers some of its energy to a $2 \omega_{A}$ and $2 k_{A}$ inertia-gravity wave ( $\mathrm{E}$ in Fig. 15); this is observed as a free wave radiating away from the beam (not shown).

The second energy transfer involves the nearsimultaneous appearance of two types of motion confined to the beam: a 36-day wave ( $B$ in Fig. 15) and a near-annual motion ( $C$ in Fig. 15$)$. The 36-day wave has little zonal variation within the beam, a $550-\mathrm{m}$ wavelength in the vertical, and the meridional structure of a Yanai wave. The near-annual motion has the same zonal wavelength as the directly forced Yanai wave, half its vertical wavelength, and a meridional structure that resembles that of a first-meridional-mode, short-wavelength Rossby wave. Its energy, however, is confined to the beam rather than radiating out along the Rossby wave ray path, suggesting that the near-annual motion is not a free wave of the system. Furthermore, the spectral peaks corresponding to motions $B$ and $C$ are related by the simple relationship

$$
\varphi_{A}=\varphi_{B}+\varphi_{C}
$$

where $\boldsymbol{\varphi}_{\mathrm{i}}=\left(\omega_{i}, k_{i}, m_{i}\right)$ is the wave vector of wave $i$ and $\omega_{i}, k_{i}$, and $m_{i}$ are its frequency, zonal, and vertical wavenumbers, respectively. Thus, the system has developed a second independent wave (degree of freedom), with the third wave then determined from the two independent ones.

Suppose that the second independent motion is wave $C$. Then, in further interactions, the wave vector $\varphi$ of each subsequent wave is determined by

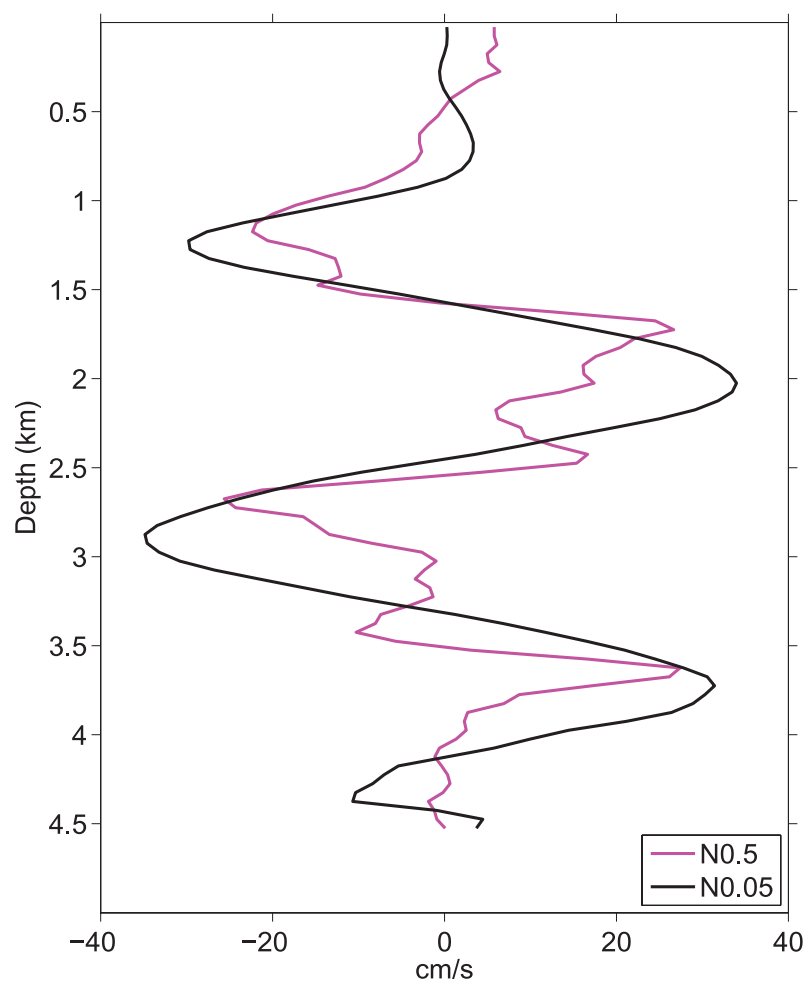

FIG. 14. Vertical profiles of equatorial $v$ on Jan 1 of model year 8 near $x=6500 \mathrm{~km}$ (within the beam) and normalized by $\tau_{0} / \tau$ in the weak (N0.05) and standard (N0.5) cases.

$$
\varphi=n \varphi_{A} \pm p \varphi_{C}
$$

where $n$ and $p$ are integers. These interactions result in the energy being distributed over bands that are parallel in the frequency-wavenumber space to the wave vector of the near-annual motion (dashed white line in Fig. 15).

An example of the chronology of these nonlinear interactions is shown in Fig. 16. After year 4, the nearannual motion and the 36-day wave (blue and magenta in Fig. 16a) start to grow on the energy of the directly forced Yanai wave (red in Fig. 16a). These and further interactions result in an increase in energy at small vertical scales and in the effective dissipation of the beam, mainly via vertical friction of horizontal momentum (Fig. 16b). This process is generally known as wave breaking and is qualitatively similar to the breaking of internal gravity waves (McEwan 1971). The dissipating waves generate EICS currents to the west of the beam after year 7 (Fig. 16c). The amplitude of the EICS stays constant up to year 14 and then increases again following an increase in the explicit dissipation (Fig. 16b). The increase in explicit dissipation may be associated with motions in the 50-100-day band that appear at that time.

In this experiment, the EICS takes about 7-10 yr to appear in the west (Fig. 16c), whereas it takes less than 


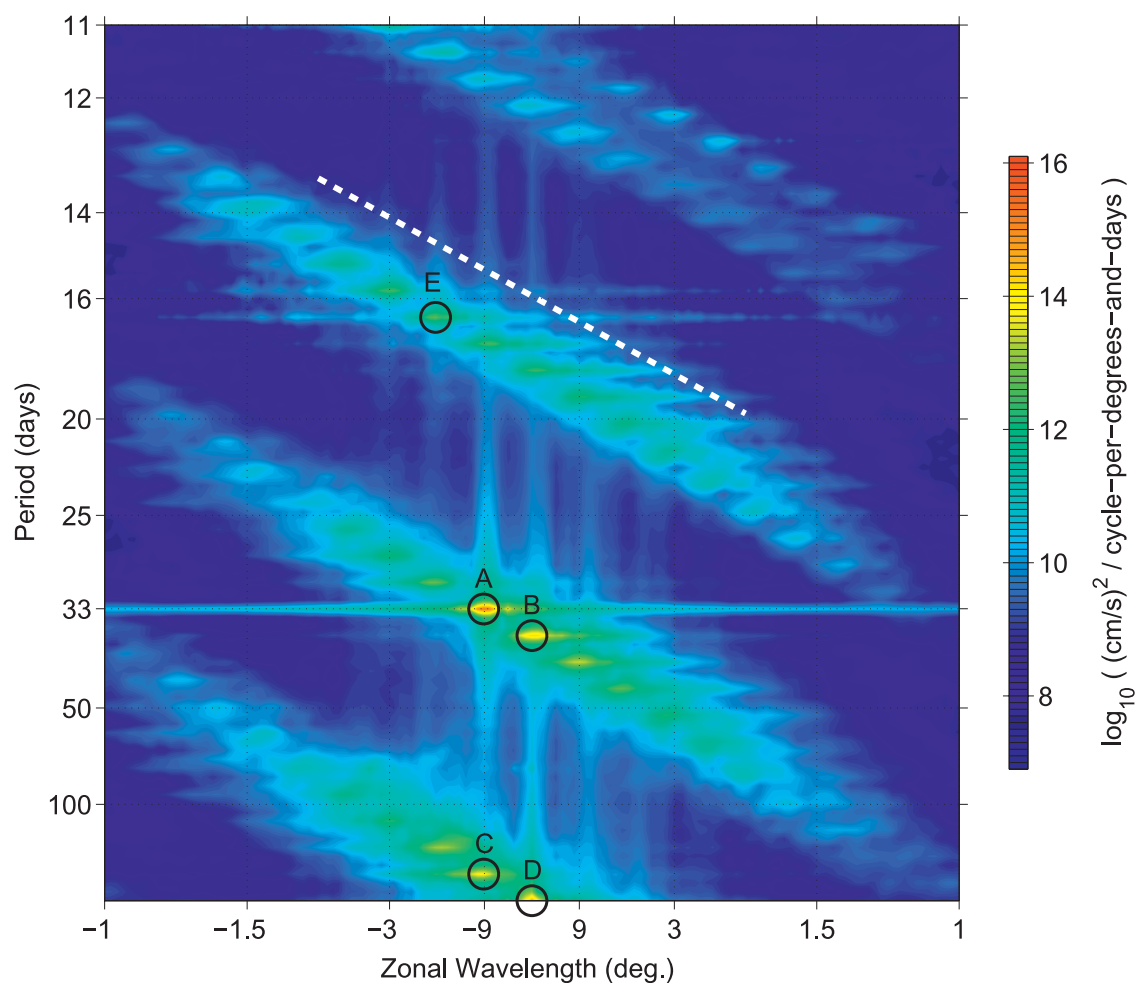

FIG. 15. Power spectrum in $k-\omega$ space averaged between 1000 and $3000 \mathrm{~m}$ of equatorial horizontal velocity over years 7-9 in the moderate case (N0.25). The spectrum is obtained by summing the spectra of $u$ and $v$. The circles indicate some of the waves that take part in the transfer of energy throughout the frequency-wavenumber space: directly forced $\omega_{A}$ Yanai wave (A), 36-day Yanai wave (B), near-annual motion (C), mean flow (D), and $2 \omega_{A}$ wave (E). The dashed white line is a parallel to the wave vector of the near-annual motion.

$3 \mathrm{yr}$ to appear in the experiments with standard and strong forcing, which is consistent with the idea that the beam breaks more rapidly with larger amplitude. Therefore, it is possible that, if the experiments with weak forcing were run longer, the breaking would eventually occur, causing dissipation of the Yanai beam. Unfortunately, we have no explanation of why the breaking of the beam starts with the near-annual motion and the 36-day wave.

As noted earlier, in the experiments with stronger forcing, the spectrum looks very much like that of the moderate case, except that energy is distributed continuously within each energy band. Such behavior, from discrete peaks at moderate amplitude to continuous energy distribution at larger amplitude, is typical of the quasi-periodic route to turbulence (Bergé et al. 1984). See McCreary and Yu (1992) for an example of a similar route to turbulence in a numerical model of the equatorial ocean.

\section{Analytical solution}

In this section, we construct an analytical solution for the Eulerian-mean flow obtained in the numerical simulations. We assume that the Yanai beam has a weak amplitude $O(\epsilon)$, where $\epsilon$ is a small parameter; it is weakly dissipated by Rayleigh friction while its energy is propagating downward and to the east, but it is persistently forced at the surface so that the amplitude $\epsilon$ is a function of depth but not of time. We assume also that there is no motion at $O(1)$ and that the Yanai beam is the only motion at $O(\epsilon)$, and we look for the time-independent solution at $O\left(\epsilon^{2}\right)$ that results from self-advection combined with dissipation of the Yanai beam.

We used two different approaches. One approach computes the Eulerian-mean flow directly. First, the Eulerian means of the advective terms resulting from the dissipated Yanai beam are calculated, and then they are used to force the Eulerian-mean solution at $O\left(\epsilon^{2}\right)$ (for details, see Ascani 2008). In this case, however, the role of dissipation in forming Eulerian-mean flow to the west of the beam is difficult to interpret physically because it depends on the changes in relative phase between wave quantities.

The second approach computes the Eulerian-mean flow $\mathbf{U}_{E}$ from the difference between the Lagrangianmean flow $\mathbf{U}_{L}$ and the Stokes drift $\mathbf{U}_{S}$; that is, 
(a)

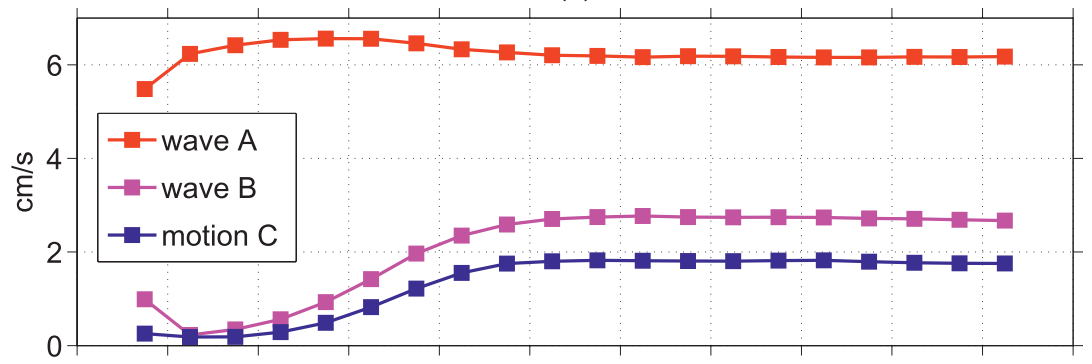

(b)

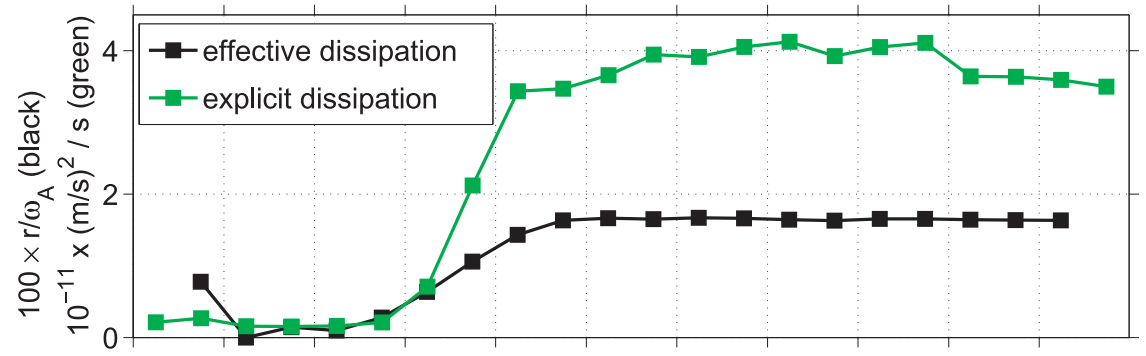

(c)

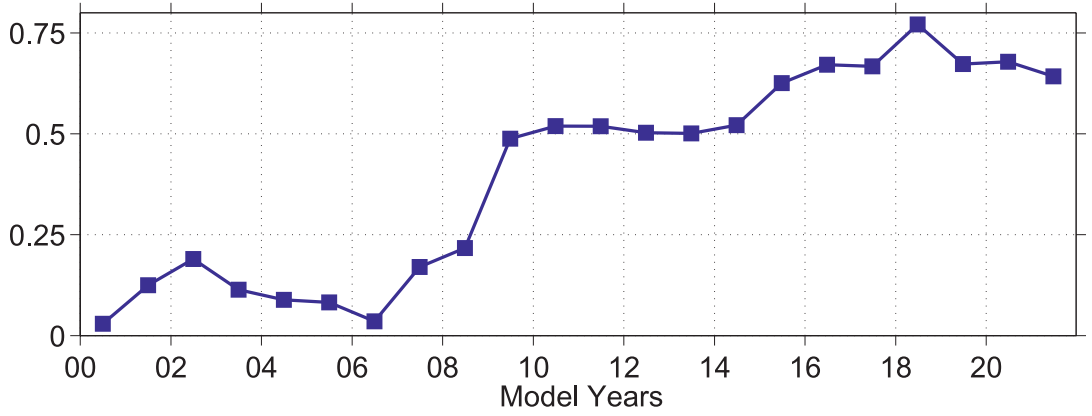

FIG. 16. Chronology of the energy cascade in the moderate case (N0.25): (a) amplitude of the equatorial horizontal velocity (square root of the sum of squared amplitude of zonal and meridional velocity) for waves A, B, and C (see Fig. 15), calculated from Fourier decomposition; (b) effective dissipation of the beam via $r / \omega_{A}$ and explicit dissipation via the work lost by vertical friction; and (c) ratio of the amplitude of the westward equatorial current (west of/ within) the beam. In all cases, the quantities are computed over 3-yr-long segments translated by a year. The work is computed at the equator and averaged along the beam between $z_{\max }$ and $z_{\text {min }}$ (see Fig. 6) and between the beam edges.

$$
\mathbf{U}_{E}=\mathbf{U}_{L}-\mathbf{U}_{S}
$$

The Lagrangian-mean flow is computed from the PV equation using the kinematic properties of a Lagrangian mean (defined later) as described by the generalized Lagrangian-mean (GLM) theory of Andrews and McIntyre (1978), whereas the Stokes drift is a purely kinematic quantity and is deduced directly from the wave field alone. This approach enables us to show explicitly the role of dissipation in the Lagrangian circulation. Furthermore, it enables us to reformulate the nonacceleration theorem for the case of time-mean flow in a rotating basin lacking closed PV contours, a version that has not been emphasized in literature. For these reasons, only the second approach is presented.

In the following, we first introduce the Eulerian-mean, Lagrangian-mean, and Stokes operators, as well as the expansion in $\epsilon$ [section $4 \mathrm{a}(1)]$. We derive the solution for the Stokes drift and Lagrangian-mean flow [sections $4 a(2)$ and $4 a(3)]$. We then discuss our analytical results, comparing them to two of our numerical solutions [section $4 \mathrm{~b}(1)$ ] and exploring their sensitivity to the central 
period and zonal wavelength of the Yanai beam [section $4 b(2)]$.

\section{a. Derivation}

\section{1) EULERIAN-MEAN, LAGRANGIAN-MEAN, AND STOKES OPERATORS}

We define the Eulerian-mean operator $\overline{()}$ for any quantity $q(\mathbf{x}, t)$ as

$$
\overline{q(\mathbf{x}, t)}=\frac{1}{T} \int_{0}^{T} q(\mathbf{x}, t) d t,
$$

where $\mathbf{x}$ is the position vector, $t$ is time, and $T$ corresponds to one wave cycle. With no diapycnal mixing, water parcels are restricted to isopycnal surfaces. For this reason, we use isopycnal coordinates: The position vector is defined as $\mathbf{x}=(x, y, \rho)$, where $x$ and $y$ are the zonal and meridional coordinates, respectively, and $\rho$ is the potential density. The Eulerian-mean flow is thus

$$
\mathbf{U}_{E}=\left(U_{E}, V_{E}\right)=(\bar{u}, \bar{v}),
$$

where $u$ and $v$ are the zonal and meridional velocity along isopycnal surfaces, respectively. In the following, the actual position of a parcel is $\mathbf{x}^{\xi}$; its Eulerian-mean position between 0 and $T$ is $\mathbf{x}$; and its displacement anomaly $\boldsymbol{\xi}=\mathbf{x}^{\bar{\xi}}-\mathbf{x}$ which assures that $\overline{\boldsymbol{\xi}}=0$. With no diapycnal mixing, the $\rho$ component of $\boldsymbol{\xi}$ is zero.

The Lagrangian-mean operator $\overline{()}^{L}$ is defined as

$$
\bar{q}^{L}=\overline{q^{\xi}},
$$

where $q^{\xi}=q(\mathbf{x}+\boldsymbol{\xi}, t)$ is the value of $q$ following a parcel that has an Eulerian-mean position $\mathbf{x}$ (Andrews and McIntyre 1978; Bühler 2009). Because $\bar{q}^{L}$ is associated with the fixed Eulerian position $\mathbf{x}$, it is not a purely Lagrangian quantity in the classic sense but is rather a hybrid EulerianLagrangian quantity. The Lagrangian-mean velocity is defined as

$$
\mathbf{U}_{L}=\left(U_{L}, V_{L}\right)=\left(\bar{u}^{L}, \bar{v}^{L}\right) .
$$

The key kinematical property of the Lagrangianmean operator is

$$
\overline{D q}^{L}=\bar{D}^{L} \bar{q}^{L}
$$

where

$$
\frac{D}{D t}=\partial_{t}+u \partial_{x}+v \partial_{y}
$$

is the derivative following the motion and

$$
\frac{\bar{D}^{L}}{D t}=\partial_{t}+U_{L} \partial_{x}+V_{L} \partial_{y}
$$

Equation (12) is the central result of the GLM theory, and we refer to Andrews and McIntyre (1978) for its derivation (see also McIntyre 1980).

The Stokes operator $\overline{()}$ is defined as

$$
\bar{q}^{S}=\bar{q}^{L}-\overline{q(\mathbf{x}, t)},
$$

and the Stokes drift is defined as

$$
\mathbf{U}_{s}=\left(U_{S}, V_{S}\right)=\left(\bar{u}^{S}, \bar{v}^{S}\right) .
$$

However, we can calculate $\bar{q}^{S}$ directly from the wave field without calculating first $\bar{q}^{L}$ and $\overline{q(\mathbf{x}, t)}$, if we expand $q$ in powers of $\epsilon$ :

$$
q(\mathbf{x}, t)=q_{0}+q_{1}+q_{2}+O\left(\epsilon^{3}\right),
$$

where $q_{n}$ is $O\left(\epsilon^{n}\right)$ and is evaluated at (x,t). In (17), $q_{0}=0$ for most quantities, because we are considering an oscillatory Yanai beam. The exceptions are for PV and the background stratification, which do have a contribution at lowest order.

Using this expansion, the Stokes operator takes the following explicit form for any quantity that has no zeroth order $\left(q_{0}=0\right)$. We start with a Taylor expansion of $q^{\xi}$ (Andrews and McIntyre 1978):

$$
q^{\xi}=q(\mathbf{x}, t)+\boldsymbol{\xi} \cdot \nabla q(\mathbf{x}, t)+O\left(\epsilon^{3}\right) .
$$

Applying the Eulerian-mean operator to (18) and using (10), (15), and the expansion (17) gives the leading order $O\left(\epsilon^{2}\right)$ of the Stokes operator in terms of wave quantities alone,

$$
\bar{q}^{S}=\overline{\boldsymbol{\xi}_{1} \cdot \nabla q_{1}}+O\left(\epsilon^{3}\right),
$$

where $\boldsymbol{\xi}_{1}$ is the first-order displacement anomaly and is calculated by integrating the wave velocity field with time,

$$
\boldsymbol{\xi}_{1}=\int_{0}^{t}\left(u_{1}, v_{1}\right)\left(\mathbf{x}, t^{\prime}\right) d t^{\prime} .
$$

\section{2) STOKES DRIFT}

According to (16) and (19),

$$
\begin{aligned}
& U_{S}=\overline{\boldsymbol{\xi}_{1} \cdot \nabla u_{1}}+O\left(\epsilon^{3}\right) \quad \text { and } \\
& V_{S}=\overline{\boldsymbol{\xi}_{1} \cdot \nabla v_{1}}+O\left(\epsilon^{3}\right) .
\end{aligned}
$$

The Stokes drift is the net displacement of water parcels over a wave cycle based on the wave field alone; it is 
purely kinematic. It is given by the correlation between the position of the water parcel and the local gradient of the wave velocity field. For an inviscid Yanai wave, this correlation is nonzero: $\xi_{1}$ is in phase with $\nabla u_{1}$ and $90^{\circ}$ out of phase with $\nabla v_{1}$ so that the Stokes drift is purely zonal. Thus, when the dissipation is weak, the changes that appear in the relative phase between wave quantities can be ignored and the Stokes drift can then be approximated by the viscid wave solution that takes into account only the decay of amplitude with depth. We have checked numerically that this is indeed true. This wave solution and the method for applying (21) and (22) to a Yanai beam are presented in the appendix.

\section{3) LAGRANGIAN-MEAN VELOCITY}

The Lagrangian-mean velocity is computed from the Lagrangian-mean PV and continuity equations. Rayleigh friction, with coefficient $r$, provides a simple model of dissipation. The momentum equations in isopycnal coordinates are then (Kasahara 1974)

$$
\begin{aligned}
& \frac{D u}{D t}-f v=-\frac{1}{\rho_{0}} \partial_{x} P-r u \quad \text { and } \\
& \frac{D v}{D t}+f u=-\frac{1}{\rho_{0}} \partial_{y} P-r v,
\end{aligned}
$$

where $f$ is the planetary vorticity and $P$ is the Montgomery potential

$$
P=p+\rho g z
$$

with $p$ the pressure anomaly. The hydrostatic equation is

$$
\partial_{\rho} P=g z
$$

and the continuity equation is

$$
\partial_{t} z_{\rho}+\partial_{x}\left(u z_{\rho}\right)+\partial_{y}\left(v z_{\rho}\right)=0
$$

where $z_{\rho}=\partial_{\rho} z$.

The PV equation is obtained by taking the curl of (23) and (24) and using (27) to get

$$
\frac{D}{D t}\left(\frac{f+\zeta}{z_{\rho}}\right)=-r \frac{\zeta}{z_{\rho}},
$$

where $\zeta=\partial_{x} v-\partial_{y} u$ is the relative vorticity. Using (12), its Lagrangian mean is simply

$$
\frac{\bar{D}^{L}}{D t}{\overline{\left[\frac{f+\zeta}{z_{\rho}}\right]}}^{L}=-r\left[\frac{\zeta}{z_{\rho}}\right]^{L} .
$$

In the following, we are looking for the lowest-order version of (29) from which we deduce the Lagrangianmean flow.

First, we approximate the lhs of (29). In the case of a uniform and constant background stratification given by its buoyancy frequency $\hat{N}$, the zeroth order of $z_{\rho}$ is $\hat{z}_{\rho}=-g /\left(\rho_{0} \hat{N}^{2}\right)$. With no $O(1)$ and $O(\epsilon)$ mean flow, then, the lowest order of the Lagrangian-mean PV reduces to the background PV: that is,

$$
\frac{\overline{f+\zeta}^{L}}{z_{\rho}}=\frac{f}{\hat{z}_{\rho}}+O(\epsilon) .
$$

With constant uniform stratification, anticipating that $\mathbf{U}_{L}$ is $O\left(\epsilon^{2}\right)$ and using definition (14),

$$
\frac{\bar{D}^{L}}{\overline{D t}}{\overline{\left[\frac{f+\zeta}{z_{\rho}}\right]}}^{L}=\frac{\beta}{\hat{z}_{\rho}} V_{L}+O\left(\epsilon^{3}\right),
$$

where $\beta=d f / d y$ is the meridional gradient of planetary vorticity.

We now approximate the rhs of (29). The expansion of the perturbation $\mathrm{PV}$ is

$$
\frac{\zeta}{z_{\rho}}=\frac{1}{\hat{z}_{\rho}}\left(\zeta_{1}+\zeta_{2}-\frac{\zeta_{1} z_{\rho_{1}}}{\hat{z}_{\rho}}\right)+O\left(\epsilon^{3}\right) .
$$

Separating the Stokes and Eulerian-mean components, the Lagrangian mean of (32) is

$$
\begin{aligned}
{\overline{\left[\frac{\zeta}{z_{\rho}}\right]}}^{L}= & \frac{1}{\hat{z}_{\rho}}\left[\overline{\zeta_{1}}+\bar{\zeta}_{1}^{S}+\bar{\zeta}_{2}+\bar{\zeta}_{2}^{S}-\frac{\left.\overline{\zeta_{1} z_{\rho_{1}}}-\frac{\overline{\zeta_{1} z_{\rho_{1}}}}{\hat{z}_{\rho}}\right]}{\hat{z}_{\rho}}\right] \\
& +O\left(\epsilon^{3}\right) .
\end{aligned}
$$

The first term on the rhs is zero because the Eulerian mean of any wave quantity $q_{1}$ is zero. The fourth and sixth terms are $O\left(\epsilon^{3}\right)$ according to (19). Defining $\zeta_{S}=\bar{\zeta}_{1}^{S}$ as the Stokes relative vorticity (i.e., the relative vorticity of a water parcel averaged over a wave cycle resulting from the presence of the wave field alone), noting that $\overline{\zeta_{2}}=\partial_{x} V_{E}-\partial_{y} U_{E}$ is the relative vorticity of the $O\left(\epsilon^{2}\right)$ Eulerian-mean flow, and using (31), the PV Eq. (29) at $O\left(\epsilon^{2}\right)$ can be written

$$
\frac{\beta}{\hat{z}_{\rho}} V_{L}=-r Q_{L}
$$

where

$$
Q_{L}=\frac{1}{\hat{z}_{\rho}}\left[\zeta_{S}+\left(\partial_{x} V_{E}-\partial_{y} U_{E}\right)-\frac{\overline{\zeta_{1} z_{\rho_{1}}}}{\hat{z}_{\rho}}\right]
$$


is the perturbation PV of a water parcel averaged over a wave cycle. Hence, the meridional component of the Lagrangian-mean flow is proportional to the dissipation coefficient times the perturbation PV.

With no diapycnal mixing and with uniform background stratification and time-independent wave amplitude $\epsilon$, the Lagrangian-mean continuity equation (Moore 1970) at $O\left(\epsilon^{2}\right)$ is

$$
\partial_{x} U_{L}+\partial_{y} V_{L}=0
$$

It then follows from (34) that the zonal Lagrangianmean flow is

$$
U_{L}(\mathbf{x}, t)=\int_{x}^{x_{\mathrm{EB}}} \partial_{y} V_{L}\left(\mathbf{x}^{\prime}, t\right) d x^{\prime},
$$

where we apply the condition that $U_{L}=0$ at an eastern boundary, $x=x_{\mathrm{EB}}$.

According to (34) and (37), without dissipation, there is no Lagrangian-mean flow so that the Eulerian-mean flow exactly cancels the Stokes drift (Moore 1970): that is,

$$
\mathbf{U}_{E}=-\mathbf{U}_{S} \text {. }
$$

With dissipation, there is necessarily a net meridional displacement of water parcels over each wave cycle, allowing a zonal Lagrangian-mean circulation in the presence of meridional barriers. It follows that only with dissipation can the mean flow appear west of the forcing region (Yanai beam). This property is consistent with our numerical solutions.

The principle behind the nonacceleration theorem valid for zonally averaged flows in a zonal channel can then be reformulated for the case of time-mean flows in a rotating basin lacking closed PV contours: no steady Lagrangian flow is possible if the waves are conservative. Notice that, in contrast to the nonacceleration theorem, the statement is about the existence of the flow, not its acceleration.

At this point, the Lagrangian-mean flow is not yet known because of the presence of $\mathbf{U}_{E}$ in (35). The first step is to replace $\mathbf{U}_{E}$ by $\mathbf{U}_{L}-\mathbf{U}_{S}$. The second step is to notice that $\mathbf{U}_{S}$ is of order zero in $\tilde{r}=r / \omega \ll 1$ (with $\omega$ being the wave frequency) and $\mathbf{U}_{L}$ is of order one according to (34) and (37). Thus, in (35), the contribution to $V_{L}$ by $\mathbf{U}_{S}$ is of order one in $\tilde{r}$, whereas the contribution by $\mathbf{U}_{L}$ is of second order. We can thus ignore the contribution by $\mathbf{U}_{L}$ so that

$$
Q_{L} \approx \frac{1}{\hat{z}_{\rho}}\left(\zeta_{S}-\left(\partial_{x} V_{S}-\partial_{y} U_{S}\right)-\frac{\overline{\zeta_{1} z_{\rho_{1}}}}{\hat{z}_{\rho}}\right),
$$

where all the terms on the rhs are calculated using the same wave solution used to compute the Stokes drift (for details, see the appendix). Once $\mathbf{U}_{L}$ has been estimated, we subtract $\mathbf{U}_{S}$ from it to obtain $\mathbf{U}_{E}$.

\section{b. Results}

\section{1) COMPARISON TO NUMERICAL EXPERIMENTS}

In this section, the analytical solution is compared to the solution obtained in the experiment with weak forcing and no beam dissipation (N0.05) and the experiment with standard forcing and weak beam dissipation (N0.5). In both cases, the amplitude of the beam and the dissipative coefficient used in the analytical solution are adjusted to fit the beam solution in the numerical experiment as explained in the appendix.

\section{(i) Case without dissipation (solution N0.05)}

The Eulerian-mean flow obtained analytically compares well to the numerical one, not only in meridional, vertical, and zonal structure but also in amplitude (Figs. $8 \mathrm{a}, \mathrm{c}, 10 \mathrm{a}, \mathrm{c})$. As explained in section $4 \mathrm{a}(3)$, because there is no dissipation and therefore no Lagrangian-mean flow, the Eulerian-mean flow is simply minus the Stokes drift induced by the Yanai wave. As a result, the EICS structure, with westward flow on the equator and eastward off the equator, is constrained to the beam (Figs. $10 \mathrm{a}, \mathrm{c})$. Furthermore, because the Stokes drift is uniform with distance along the beam, so are the analytical EICS currents and so are the numerical EICS currents over the depth range that is not influenced by boundary effects (Figs. 8a,c).

\section{(ii) Case with dissipation (solution N0.5)}

With dissipation, the Eulerian-mean flow extends west of the forcing region (Fig. 10b), an indication that the Lagrangian-mean flow is nonzero. In (34) and (39), the Yanai wave produces equatorward contributions from $\zeta_{S}$ and $-\overline{\zeta_{1} z_{\rho_{1}}}$ that are only partially compensated by $-\left(\partial_{x} V_{S}-\partial_{y} U_{S}\right)$. This equatorward flow within the beam is supplied by off-equatorial eastward flows from the western boundary and in turn feeds an equatorial westward flow that extends from the beam to the western boundary. The meridional structure of $U_{L}$ is similar to that of $-U_{S}$, so they add constructively within the beam, where $U_{E}$ is strongest.

The analytical EICS currents resemble the numerical ones in vertical scale (Figs. 8b,d) and in zonal and meridional structure (Figs. 10b,d). Although they are as strong as the numerical EICS currents west of the beam, they are weaker within the beam: $25 \%$ weaker for the westward equatorial jet and 50\% weaker for the eastward ones. Furthermore, the analytical solution does not 
predict the off-equatorial westward flows found in the numerical experiment. In the analytical solution with constant dissipation, the amplitude of the EICS increases quadratically with the beam amplitude. The numerical solutions display roughly this behavior within the beam, but for the EICS amplitude west of the beam (Fig. 11) there seems to be a Yanai wave amplitude threshold below which the Lagrangian flow is very weak.

In the analytical solution, the ratio of the amplitude of the EICS currents west of the beam to the amplitude in the beam depends on the dissipation but not on the beam amplitude: the stronger the dissipation, the stronger the flows west of the beam relative to those within the beam (Fig. 13). However, the fit between the numerical solutions and the analytic prediction of this ratio is not good: in the numerical solutions the ratio stays roughly constant as dissipation is increased by a factor of 3 .

\section{2) SENSITIVITY TO PERIOD AND ZONAL WAVELENGTH}

The amplitude and meridional profile of the Lagrangianmean zonal flow vary with the Yanai wave period and wavenumber (Fig. 17). The amplitude of the EICS currents increases with the nonlinearity $M$, defined in (2), of the Yanai wave. When the period increases for constant wavelength and $V$ amplitude, the Yanai wave becomes more nonlinear and the EICS is stronger. On the other hand, the EICS will decay more rapidly with depth (not shown), because the vertical group velocity of the beam decreases with increasing period. For constant frequency and $V$ amplitude, there is a zonal wavelength at which $M$ is a maximum, hence the maximum EICS currents for intermediate wavelengths in Fig. 17b. In both cases (holding either the zonal wavelength or the period constant and increasing the other), the meridional scale of the EICS currents decreases along with the meridional and vertical scales of the Yanai beam [see (A7)].

\section{Discussion and conclusions}

Synoptic meridional sections of velocity measurements, together with averaged zonal velocity at $1000 \mathrm{~m}$ from ARGO floats (Fig. 2), show that the EICS is a basinscale feature in the Atlantic and Pacific Oceans. Given their large vertical scale and their temporal persistence, the EICS currents must be the zonal limbs of meridionally narrow recirculation cells; hence, they must be generated by slow meridional flow across mean PV isolines in the interior or eastern portions of the basins. The central question addressed in this paper is, what is responsible for systematically changing the $\mathrm{PV}$ of water parcels over $O(2000 \mathrm{~m})$ depth range below the thermocline in the equatorial Atlantic and Pacific? Inspired by the large body of work on mean-flow generation by waves (e.g., Moore 1970; Andrews and McIntyre 1976; McIntyre and Norton 1990; Bühler 2009), we hypothesize that the agent of PV modification is the dissipation of waves propagating through the region. We explore this hypothesis by studying a type of wave that is prominent in the spectra of the few available moored current meter measurements and numerical simulations, namely monthly periodic Yanai waves, generated by instability of the upper-ocean circulation, that form a beam of energy radiating down and to the east.

Idealized numerical simulations and an even more idealized analytic model show that such a Yanai wave beam does indeed produce a mean velocity structure resembling that of the EICS within and - more importantlyto the west of the beam. The Eulerian-mean flow in the beam is the sum of the Lagrangian-mean flow and a component canceling the Stokes drift; however, to the west of the beam, the Eulerian and Lagrangian means are identical. The Lagrangian-mean meridional flow within the beam can cross the mean PV isolines, because the perturbation PV of a water parcel averaged over a wave cycle is nonzero; any dissipation that is proportional to this yields a net change in PV over the cycle. In the analytic model the dissipation is Rayleigh friction; in the numerical simulation, for sufficient amplitude, the Yanai waves break, moving energy to higher vertical wavenumbers and lower frequencies until it is removed by explicit friction, primarily vertical. In spite of this difference in the dissipation mechanism, the analytic model and the numerical simulations produce similar mean flows.

The present theory complements the recent works of D07, H08, and M09. In those studies, the EICS currents are seen as long Rossby waves resulting from inviscid nonlinear interactions. Those works thus focus on the initial formation of the EICS. The present study focuses rather on the final stage of the EICS where they are part of a steady circulation. For this circulation to exist in a basin, irreversible changes of PV are necessary. Hence, dissipation must be an essential element of any mean recirculations generated by the numerical simulations of D07, H08, and M09. Their work and our work are similar in focusing on intraseasonal Yanai waves but differ in the source and characteristics of the Yanai waves and in the relation between the Yanai wave structure and the meridional structure of the resulting mean currents. In their model, the structure of the mean currents is that of a long Rossby wave growing from an instability; in our model, it is a direct consequence of the structure of the primary Yanai wave itself. 
(a) Sensitivity to wave period

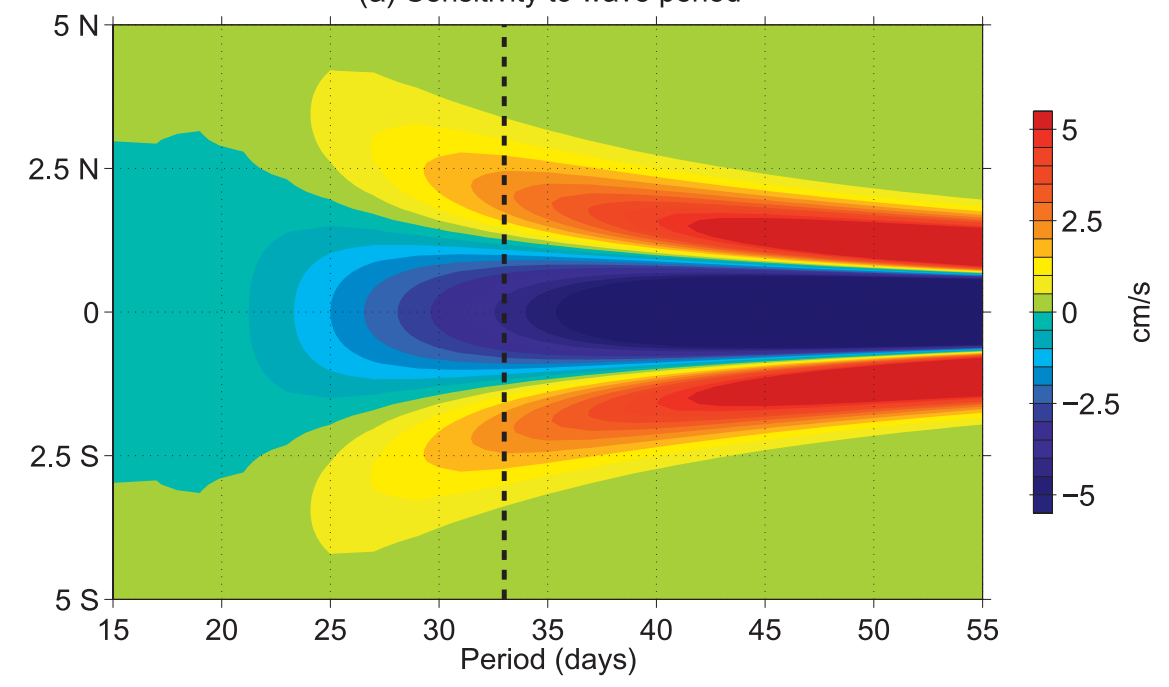

(b) Sensitivity to zonal wavelength

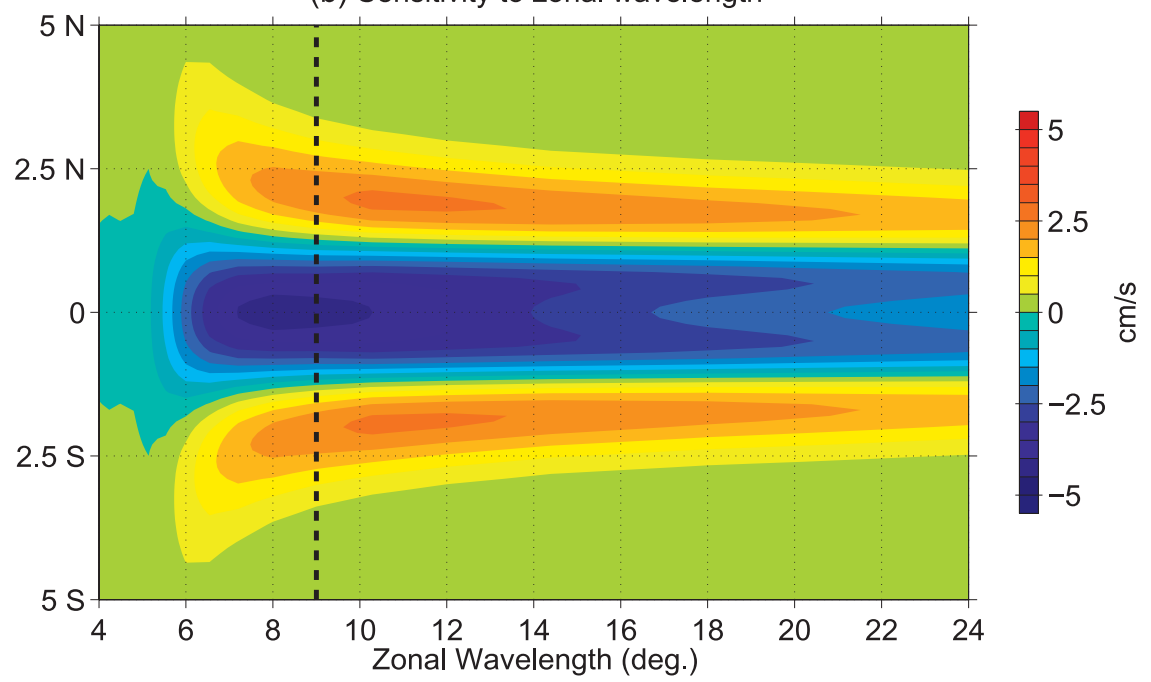

FIG. 17. Sensitivity of the Lagrangian-mean zonal flow at $x=0$ (west of beam) and $\rho=0$ to (a) the central period and (b) central zonal wavelength of the Yanai beam. All other values are those used for the analytical solution with dissipation [section $4 \mathrm{~b}(1)$ (ii); shown by the black dashed lines], except that the value of $\epsilon_{0}$ is adjusted to keep the absolute amplitude $c \epsilon_{0}$ constant.

Although the present model generates meridionally alternating mean zonal flows with some resemblance to the EICS currents and provides a possible explanation for the large zonal extent of the EICS in the Pacific, it has shortcomings. First, it does not address the EDJs. Second, the mechanism seems to be too weak; in our idealized simulations as in OGCMs, to approach realistic amplitudes of the EICS the model must have a stronger Yanai beam amplitude than the sparse observations indicate. Third, the model produces little structure poleward of the eastward currents on either side of the equator; in contrast, the observations show strong westward flows poleward of these eastward currents and perhaps a continuation of the alternating pattern to even higher latitudes as in Fig. 2. We speculate that the basic mechanism in our model-systematic PV modification as a side effect of the dissipation of equatorially trapped waves - may be correct but may involve a broader spectrum of waves than we have considered so far.

The atmospheric quasi-biennial oscillation (QBO; for a review, see Baldwin et al. 2001) provides an interesting point of comparison: it consists of eastward and westward zonal flows in the equatorial stratosphere, alternating in time, propagating downward with a period slightly longer than $2 \mathrm{yr}$, and driven by upward-propagating highfrequency equatorially trapped waves. The mechanism 
by which these waves drive the QBO is also wave dissipation (Holton and Lindzen 1972). The QBO mechanism is based on two-way wave-mean-flow interaction, whereas our interaction is a one-way interaction; the decaying waves drive the mean flow without being greatly modified by it. Nevertheless, we find an interesting parallel between studies of the QBO and those of the EICS (and EDJs): in both cases, realistic numerical simulations have been difficult to achieve. Baldwin et al. (2001) summarize this in the following:

The most important factor in reproducing the QBO is probably the use of a fine vertical resolution to resolve equatorial gravity waves. The horizontal diffusion should be weak enough not to prevent the evolution of the mean flow oscillation and not to be the primary damping mechanism for the waves. Transient characteristics of tropical cumulus convection are also important since they determine the excitation of the waves. Despite the recent success, those models that reproduced the QBO might have overly active cumulus convection and hence excessively large amplitude of gravity waves with resolved scales.

In the case of the EICS and the EDJs as well, it seems that better results are obtained when the model is driven with stronger than realistic winds, as in the Pacific basin of the I98 model (Figs. 3, 4). We conclude that a toostrong large-scale forcing is needed to overcome as yet unidentified deficiencies in model physics and/or forcing. Resolution alone is not the key; simulations with up to 400 levels and $1 / 8^{\circ}$ resolution (not shown here) did not substantially strengthen the EICS. Other factors could be the numerical code itself, especially the advective scheme that may not conserve PV, or small-scale phenomena such as inertia-gravity waves that are neglected in OGCM simulations but may contribute to the PV dissipation (Muench and Kunze 2000).

Another shortcoming of the present study is that the processes by which the Yanai waves break, in particular the formation of the near-annual motion and the 36-day wave, have not been elucidated. Such near-annual motion is not observed in the simulations of D07, H08, and M09, suggesting that the present instability is inherently different from that obtained in these previous studies. Interestingly, the near-annual motion also appears in another set of simulations, not presented here, in which off-equatorial forcing is used to generate barotropic and monthly periodic Yanai waves such as those produced by the instabilities of the North Equatorial Countercurrent.

In addition to modeling and theoretical issues, the present work raises questions that can only be addressed with additional observations. We need to know more about the spatial structure and temporal variability of Yanai and other waves in the subthermocline Pacific and
Atlantic. We would also benefit from long-term Lagrangian measurements of the EICS, showing where and when net meridional displacements occur.

Acknowledgments. F. Ascani and Eric Firing gratefully acknowledge the support of the National Science Foundation (NSF) through Grant OCE0327334. P. Dutrieux also acknowledges the support of NSF through Grant OCE0550857. J. P. McCreary is supported by the Japan Agency for Marine-Earth Science and Technology (JAMSTEC) through its sponsorship of the International Pacific Research Center (IPRC). This study uses the YoMaHa'07 (Lebedev et al. 2007) dataset of velocities derived from ARGO float trajectories provided by APDRC/IPRC. The I98 model simulation was conducted on the NEC SX computer of JAMSTEC. The OFES simulation was conducted on the Earth Simulator under the support of JAMSTEC. We also thank the IPRC for their computer facilities as well as the staff for their technical support. Thanks to R. H. Weisberg and L. Bunge for sharing their deep velocity measurements of the Pacific and Atlantic Oceans. Many thanks also to D. Moore, D. S. Luther, R. Furue, A. Natarov, and R. Lukas for enlightening comments throughout the preparation of this manuscript. Finally, we would like to acknowledge the reviewers for their comments; we are particularly grateful to one reviewer for pointing out the relevance of wave breaking and for clarifying some subtleties of the generalized Lagrangian-mean theory.

\section{APPENDIX}

\section{Beam Solution}

In this appendix, we show how we construct the Yanai wave beam that is used in section 4 . We first write down the solution for a single wave, and then we form a beam from a packet of individual waves. Finally, we discuss how the Stokes drift $\mathbf{U}_{S}$ and the terms in (39) required to calculate $Q_{L}$ and ultimately $\mathbf{U}_{L}$ are computed in practice.

\section{a. Waves for an individual $k$}

The individual wave solution of (23)-(27) for a single period $\omega$ and real zonal wavenumber $k$ is

$$
\begin{aligned}
v_{1} & =c \epsilon(\rho) \Re\left(\hat{v}_{\varphi}\right), \\
u_{1} & =c \epsilon(\rho) \Re\left[(i / c) \omega\left(y \hat{v}_{\varphi}\right)\right], \\
P_{1} / \rho_{0} & =c \epsilon(\rho) \Re\left[i \omega\left(y \hat{v}_{\varphi}\right)\right], \quad \text { and } \\
z_{1} / \rho_{0} & =c \epsilon(\rho) \Re\left[-\frac{m^{r}}{g \hat{\rho}_{z}} \omega\left(y \hat{v}_{\varphi}\right)\right],
\end{aligned}
$$


where

$$
\begin{aligned}
\hat{v}_{\varphi} & =e^{-\frac{\beta}{2 c} y^{2}} e^{i\left[k x+m^{r}\left(\rho / \hat{\rho}_{z}\right)-\omega t+\varphi\right]}, \\
\epsilon(\rho) & =\epsilon_{0} e^{-m^{i}\left(\rho / \hat{\rho}_{z}\right)},
\end{aligned}
$$

and $\varphi$ is the phase of the wave at $(x, \rho, t)=(0,0,0)$. The vertical wavenumber, $m=m^{r}+i m^{i}$, determined from the dispersion relation is

$$
m= \pm \hat{N} \frac{k+\frac{\beta}{\omega+i r}}{\sqrt{\omega(\omega+i r)}} .
$$

In this solution, only the effect of dissipation on the local wave amplitude $\epsilon(\rho)$, through $m^{i}$, is kept. The effect of dissipation on the relative phase between wave quantities has not been included. We have checked numerically that, for weak dissipation, this additional effect is indeed not necessary to illustrate the dynamics of the EICS. This is one of the several advantages of obtaining the Eulerian-mean flow indirectly by calculating the Stokes drift and the Lagrangian-mean flow.

The sign in (A7) determines whether the wave phase propagates upward or downward. With $\rho / \hat{\rho}_{z}$ increasing upward ( $z$ positive up), the phase of the wave propagates upward for $m^{r}<0$. In the following, only waves with upward phase propagation (downward energy propagation) are considered and the + sign is taken. For those waves, $c=\hat{N} / m^{r}$ is the gravity wave speed of the inviscid case, and according to (A5) the meridional profile of $v$ is a Gaussian centered on the equator.

\section{b. Construction of a beam}

To form a beam, a sum of wave solutions of the form (A1)-(A4) is used. The sum is performed over a finite number of waves with frequency, wavenumbers, and phase $\boldsymbol{\varphi}_{n}=\left(\omega_{A}, k_{n}, m_{n}, \varphi_{n}\right)$; vertical profile $\epsilon_{n}(\rho)=$ $\epsilon_{0, n} e^{-m_{n}^{i}\left(\rho / \hat{\rho}_{z}\right)}$; and velocity scale $c_{n}$, with $n$ being the index of each wave and $\omega_{A}$ being the beam central frequency. For each $k_{n}$, the vertical wavenumber $m_{n}$ is computed using (A7). The beam solution used in section 4 is then

$$
v_{1}=\sum_{n} v_{n, 1}=\sum_{n} c_{n} \epsilon_{n}(\rho) \Re\left(\hat{v}_{\varphi_{n}}\right),
$$

and similarly $u_{1}=\sum_{n} u_{n, 1}$, etc. The relative magnitude of each wave, $\left(c_{n} \epsilon_{0, n}\right) /\left(c_{A} \epsilon_{A, 0}\right)$, and the wave properties $\left(k_{n}, \varphi_{n}\right)$ are obtained from the Fourier decomposition in $x$ of the zonal profile $X(x)$ of the surface forcing used to generate the Yanai beam in the numerical experiments (section 3a).

To estimate the last two parameters, the overall normalized amplitude $\epsilon_{A, 0}$, and the Rayleigh friction coefficient $r$, the wave field within the beam is approximated by a single wave with $\varphi_{A}=\left(\omega_{A}, k_{A}, m_{A}, \varphi_{A}\right)$, velocity scale $c_{A} \approx 53 \mathrm{~cm} \mathrm{~s}^{-1}$, and a vertical profile $\epsilon_{A}(\rho)$ so that

$$
v_{1} \approx c_{A} \epsilon_{A}(\rho) \Re\left(\hat{v}_{\varphi_{A}}\right),
$$

where $\epsilon_{A}(\rho)=\epsilon_{A, 0} e^{-m_{A}^{i}\left(\rho / \hat{\rho}_{z}\right)} ; \epsilon_{A, 0}$ and $m_{A}^{i}$ are deduced by fitting $c_{A} \epsilon_{A}(\rho)$ to the profile $V$ of the meridional velocity at the equator obtained within the beam in each numerical simulation as explained in section 3c. An estimate of $r$ is then obtained numerically from $m_{A}^{i}, \omega_{A}$, and $k_{A}$ using the dispersion relation (A7).

An example of the analytical beam solution constructed to fit the numerical beam solution in the standard case (N0.5) is shown in Fig. 6b. Depths below about $2250 \mathrm{~m}$ are not shown, because they correspond to the portion of the water column where the beam reaches the eastern boundary and the analytical solution is incomplete.

\section{c. Calculation of $\mathbf{U}_{S}$ and $Q_{L}$}

To compute the Stokes drift $\mathbf{U}_{S}$ as well as the terms in (39) to deduce $Q_{L}$ and the Lagrangian-mean flow, we need to calculate various correlations between wave quantities for all possible pairs of waves forming the beam. For instance, for the zonal Stokes drift,

$$
U_{S}=\sum_{n, p} \overline{\xi_{n, 1} \cdot \nabla u_{p, 1}}
$$

where the sum is performed over all pairs $(n, p)$ of waves. Individual terms in (A10) are calculated analytically, but the sum is computed numerically. This procedure is applied to deduce $V_{S}$ and all terms in (39).

\section{REFERENCES}

Andrews, D. G., and M. E. McIntyre, 1976: Planetary waves in horizontal and vertical shear: The generalized Eliassen-Palm relation and the mean zonal acceleration. J. Atmos. Sci., 33, 2031-2048.

- , and - 1978: An exact theory of nonlinear waves on a Lagrangian-mean flow. J. Fluid Mech., 89, 609-646.

Ascani, F., 2008: Wave-induced deep equatorial ocean circulation. Ph.D. thesis, University of Hawaii at Manoa, $158 \mathrm{pp}$.

Baldwin, M. P., and Coauthors, 2001: The quasi-biennial oscillation. Rev. Geophys., 39, 179-229.

Bergé, P., Y. Pomeau, and C. Vidal, 1984: Order within Chaos. John Wiley \& Sons, 329 pp. 
Bourlès, B., M. d'Orgeville, G. Eldin, Y. Gouriou, R. Chuchla, Y. du Penhoat, and S. Arnault, 2002: On the evolution of the thermocline and subthermocline eastward currents in the equatorial Atlantic. Geophys. Res. Lett., 29, 1785, doi:10.1029/ 2002 GL015098.

_ - and Coauthors, 2003: The deep currents in the eastern equatorial Atlantic Ocean. Geophys. Res. Lett., 30, 8002, doi:10.1029/ 2002GL015095.

Boyd, J. P., 1976: The noninteraction of waves with the zonally averaged flow on a spherical Earth and the interrelationships of eddy fluxes of energy, heat and momentum. J. Atmos. Sci., 33, 2285-2291.

Brandt, P., V. Hormann, B. Bourlès, J. Fischer, F. A. Schott, L. Stramma, and M. Dengler, 2008: Oxygen tongues and zonal currents in the equatorial Atlantic. J. Geophys. Res., 113, C04012, doi:10.1029/2007JC004435.

Bühler, O., 2009: Waves and Mean Flows. Cambridge University Press, $370 \mathrm{pp}$.

Bunge, L., C. Provost, J. M. Lilly, M. d'Orgeville, A. Kartavtseff, and J.-L. Melice, 2006: Variability of the horizontal velocity structure in the upper $1600 \mathrm{~m}$ of the water column on the equator at $10^{\circ}$ W. J. Phys. Oceanogr., 36, 1287-1304.

,-- B. L. Hua, and A. Kartavtseff, 2008: Variability at intermediate depths at the equator in the Atlantic Ocean in 2000-06: Annual cycle, equatorial deep jets, and intraseasonal meridional velocity fluctuations. J. Phys. Oceanogr., 38, 1794-1806.

Cox, M. D., 1980: Generation and propagation of 30-day waves in a numerical model of the Pacific. J. Phys. Oceanogr., 10, $1168-1186$.

d'Orgeville, M., B. L. Hua, and H. Sasaki, 2007: Equatorial Deep Jets triggered by a large vertical scale variability within the western boundary layer. J. Mar. Res., 65, 1-25.

Dunkerton, T., 1980: A Lagrangian mean theory of wave, meanflow interaction with applications to nonacceleration and its breakdown. Rev. Geophys. Space Phys., 18, 387-400.

Eriksen, C. C., 1985: Moored observations of deep low-frequency motions in the central Pacific Ocean: Vertical structure and interpretation as equatorial waves. J. Phys. Oceanogr., 15, $1085-1113$

— , and J. G. Richman, 1988: An estimate of equatorial wave energy flux at 9-and 90-day periods in the central Pacific. J. Geophys. Res., 93, 15 455-15 466.

Firing, E., 1987: Deep zonal currents in the central equatorial Pacific. J. Mar. Res., 45, 791-812.

_- S. Wijffels, and P. Hacker, 1998: Equatorial subthermocline currents across the Pacific. J. Geophys. Res., 103, 21413 21423.

Gill, A. E., 1974: The stability of planetary waves on an infinite beta-plane. Geophys. Fluid Dyn., 6, 29-47.

- 1982: Atmosphere-Ocean Dynamics. Academic Press, $662 \mathrm{pp}$.

Gouriou, Y., B. Bourlès, H. Mercier, and R. Chuchla, 1999: Deep jets in the equatorial Atlantic Ocean. J. Geophys. Res., 104, $21217-21226$.

_- and Coauthors, 2001: Deep circulation in the equatorial Atlantic Ocean. Geophys. Res. Lett., 28, 819-822.

Harrison, D., 1989: On climatological monthly mean wind stress and wind stress curl fields over the World Ocean. J. Climate, 2, 57-70.

Harvey, R. R., and W. C. Patzert, 1976: Deep current measurements suggest long waves in the eastern equatorial Pacific. Science, 193, 883-885.
Holton, J. R., and R. S. Lindzen, 1972: An updated theory for the quasi-biennial cycle of the tropical stratosphere. J. Atmos. Sci., 29, 1076-1080.

Hua, B. L., M. d'Orgeville, M. D. Fruman, C. Ménesguen, R. Schopp, P. Klein, and H. Sasaki, 2008: Destabilization of mixed Rossby gravity waves and the formation of equatorial zonal jets. J. Fluid Mech., 610, 311-341.

Ishida, A., Y. Kashino, H. Mitsudera, N. Yosioka, and T. Kadokura, 1998: Preliminary results of a global high-resolution GCM experiment. J. Fac. Sci. Hokkaido Univ., 11, 441-460.

Kasahara, A., 1974: Various vertical coordinate systems used for numerical weather prediction. Mon. Wea. Rev., 102, 509-522.

Lebedev, K. V., H. Yoshinari, N. A. Maximenko, and P. W. Hacker, 2007: YoMaHa'07: Velocity data assessed from trajectories of Argo floats at parking level and at the sea surface. IPRC Tech. Note 4, 16 pp.

Li, X., and P. Chang, 1999: Mixing induced by the Atlantic equatorial wave activity in an eddy-resolved OGCM. J. Geophys. Res., 104, 13 303-13 315.

Lyman, J. M., G. C. Johnson, and W. S. Kessler, 2007: Distinct 17- and 33-day tropical instability waves in subsurface observations. J. Phys. Oceanogr., 37, 855-872.

Maltrud, M. E., and J. L. McClean, 2005: An eddy resolving global $1 / 10^{\circ}$ ocean simulation. Ocean Modell., 8, 31-54.

Masina, S., and S. G. H. Philander, 1999: An analysis of tropical instability waves in a numerical model of the Pacific Ocean. 1. Spatial variability of the waves. J. Geophys. Res., 104, 29613 29635.

Masumoto, Y., and Coauthors, 2004: A fifty-year eddy-resolving simulation of the world ocean-preliminary outcomes of OFES (OGCM for the Earth Simulator). J. Earth Simul., 1, 35-56.

McCreary, J. P., 1984: Equatorial beams. J. Mar. Res., 42, 395-430.

— , and Z. Yu, 1992: Equatorial dynamics in a 2 1/2-layer model. Prog. Oceanogr., 29, 61-132.

McEwan, A. D., 1971: Degeneration of resonantly-excited standing internal gravity waves. J. Fluid Mech., 50, 431-448.

McIntyre, M. E., 1980: Towards a Lagrangian-mean description of stratospheric circulations and chemical transports. Philos. Trans. Roy. Soc. London, 296A, 129-148.

— stratosphere. Nature, 305, 593-600.

, and - 1984: The 'surf zone' in the stratosphere. J. Atmos. Terr. Phys., 46, 825-849.

, and - 1985: General concept of wave breaking for Rossby and gravity waves. Pure Appl. Geophys., 123, 964-975.

- and W. A. Norton, 1990: Dissipative wave-mean interactions and the transport of vorticity or potential vorticity. J. Fluid Mech., 212, 403-435.

Ménesguen, C., B. L. Hua, M. D. Fruman, and R. Schopp, 2009: Dynamics of the combined extra-equatorial and equatorial deep jets in the Atlantic. J. Mar. Res., 67, 323-346.

Menkes, C. E., and Coauthors, 2002: A whirling ecosystem in the equatorial Atlantic. Geophys. Res. Lett., 29, 1553, doi:10.1029/ 2001GL014576.

Moore, D. W., 1970: The mass transport velocity induced by free oscillations at a single frequency. Geophys. Fluid Dyn., 1, 237-247.

Muench, J. E., and E. Kunze, 2000: Internal wave interactions with equatorial deep jets. Part II: Acceleration of the jets. J. Phys. Oceanogr., 30, 2099-2110.

Ollitrault, M., M. Lankhorst, D. Fratantoni, P. Richardson, and W. Zenk, 2006: Zonal intermediate currents in the equatorial Atlantic Ocean. Geophys. Res. Lett., 33, L05605, doi:10.1029/ 2005GL025368. 
Pacanowski, R. C., and S. G. H. Philander, 1981: Parameterization of vertical mixing in numerical models of tropical oceans. J. Phys. Oceanogr., 11, 1443-1451.

Schott, F. A., L. Stramma, and J. Fischer, 1995: The warm water inflow into the western tropical Atlantic boundary regime, spring 1994. J. Geophys. Res., 100, 24 745-24 760.

— $35^{\circ} \mathrm{W}$ in the tropical Atlantic. Geophys. Res. Lett., 30, 1349, doi:10.1029/2002GL016849.

Tang, T. Y., R. H. Weisberg, and D. Halpern, 1988: Vertical structure of low frequency variability in the eastern equatorial Pacific Ocean. J. Phys. Oceanogr., 18, 1009-1019. von Schuckmann, K., P. Brandt, and C. Eden, 2008: Generation of tropical instability waves in the Atlantic Ocean. J. Geophys. Res., 113, C08034, doi:10.1029/2007JC004712.

Weisberg, R. H., and A. M. Horigan, 1981: Low-frequency variability in the equatorial Atlantic. J. Phys. Oceanogr., 11, 913-920.

—_ — , and C. Colin, 1979: Equatorially trapped Rossbygravity wave propagation in the Gulf of Guinea. J. Mar. Res., 37, 67-86.

Yu, J.-Y., and W. T. Liu, 2003: A linear relationship between ENSO intensity and tropical instability wave activity in the eastern Pacific Ocean. Geophys. Res. Lett., 30, 1735, doi:10.1029/ 2003GL017176. 